\title{
Efficient Content Delivery in the Presence of Impatient Jobs
}

\author{
M. Larrañaga ${ }^{1,5}$, O. J. Boxma ${ }^{3}$, R. Núñez-Queija ${ }^{2}$, M. S. Squillante ${ }^{4}$ \\ ${ }^{1}$ CNRS, LAAS, 7 avenue du colonel Roche, F-31400 Toulouse, France \\ ${ }^{2}$ CWI, P.O. Box 94079, 1090 GB Amsterdam, The Netherlands \\ ${ }^{3}$ Eurandom, Eindhoven University of Technology, P.O. Box 513, Eindhoven, The Netherlands \\ ${ }^{4}$ Mathematical Sciences Department, IBM Research, Yorktown Heights, NY 10598, USA \\ ${ }^{5}$ Univ. de Toulouse, INP, LAAS, F-31400 Toulouse, France
}

\begin{abstract}
We consider a content delivery problem in which jobs are processed in batches and may abandon before their service has been initiated. We model the problem as a Markovian single-server queue and analyze two different settings: (1) the system is cleared as soon as the server is activated, i.e., service rate $\mu=\infty$, and (2) the service speed is exponentially distributed with rate $\boldsymbol{\mu}<\infty$. The objective is to determine the optimal clearing strategy that minimizes the average cost incurred by holding jobs in the queue, having jobs renege, and performing setups. This last cost is incurred upon activation of the server in the case $\boldsymbol{\mu}=\infty$, and per unit of time the server is active otherwise. Our first contribution is to prove that policies of threshold type are optimal in both frameworks. In order to do so we have used the Smoothed Rate Truncation method which overcomes the problem arising from unbounded transition rates. For our second contribution, we derive the steady-state job-length distribution under threshold policies. The latter yields a characterization of the optimal threshold strategy, which can be easily implemented. Finally, we present numerical results for our solution across a wide range of parameters. We show that the performance of nonoptimal threshold policies can be very poor, which highlights the importance of computing the optimal threshold.
\end{abstract}

\section{INTRODUCTION}

In large-scale, high-volume content-delivery and filesharing networks, bandwidth resources are scarce and must be efficiently used. As the bulk of traffic is delay tolerant (e.g., software updates, video content), such requests for content can be delayed and grouped, so as to be transmitted in multi-cast mode through the network. After a request has been received, it may be better to postpone the actual transmission until one or more additional requests for the same content arrive, which may save tremendous transmission capacity. The challenge is to balance these gains against the risk of not meeting the deadline of one or more jobs.

In this paper we investigate a system that combines batch services with abandonment of jobs. Unlike typical deadlinebased models, abandonments can not be scheduled. The mechanism of abandonment, however, may be a good approximation

The PhD fellowship of Maialen Larrañaga is funded by a research grant of the Foundation Airbus Group (http://fondation.airbus-group.com/).

The research visit of Maialen Larrañaga in CWI and EURANDOM has been funded by EDSYS and SMI.

The research of Onno Boxma is funded via the NETWORKS program of the Dutch government. to deadline expiration, particularly in high-volume networks where it may require a prohibitive amount of overhead administration to monitor all individual deadlines.

Our model consists of an M/M/1 queue with an adapted service process in which jobs may be delayed for batching of service. We consider two different settings: (1) the system is cleared immediately when a batch is taken into service, and (2) the service time is exponentially distributed with positive mean $1 / \mu$. The service time of a batch is independent of the number of jobs in the batch (multi-cast). Delays due to batching come at the cost of abandonment. In particular, jobs may abandon the system while waiting to be served (expiration of their deadlines), for which we penalize the system at a fixed cost per abandoning job. Such penalties can either represent the loss of the job or the cost of serving the job on an expensive back-up service. The abandonment process is modeled assuming exponential expiration times for individual jobs. A methodology similar to ours was adopted in [1] to investigate a system in which jobs are batched for service to avoid a service set-up cost, but still must be served individually.

The objective is to minimize the average cost incurred by the system due to job waiting and abandonment as well as set up paid upon activation of the server when $\mu=\infty$ and per unit of time the server is active when $\mu<\infty$. From the perspective of abandonments it is wise to serve jobs in small batches; but it is profitable to accumulate jobs since service comes at a very high cost. Our goal is to find the optimal balance between this service and abandonment cost trade-off.

In our first contribution we explore the optimality of monotone policies, which in this setting reduces to threshold-based policies. The presence of abandonments in the queue causes the system to be non uniformizable, and hence proving optimality of monotone policies becomes extremely challenging. In a recent work [2], the authors have been able to overcome this issue by solving a truncated version of the original problem and taking the limit as the truncating parameter grows to infinity. This method has successfully been applied in [2] for a retrial queue and in [3] for a multiclass abandonment queue. In our second contribution we analyze the stationary behavior of the system and compute the steady-state job-length distribution for all $\mu \in \mathbb{R}_{+} \cup\{\infty\}$, using a generating function approach. The latter allows us to find the minimum set-up cost, for each possible state of the system, such that taking 
a batch to service or staying idle is equally appealing costwise. For the unlimited service speed case, we have been able to completely characterize the optimal threshold. In the limited service speed case, the analysis is more involved and a complete characterization could not be proven; instead the optimal threshold is determined by a function that is subject to optimization.

The remainder of the paper is structured as follows. Section II presents related work. Section III describes the model under study. In Section IV we prove that policies of threshold type are an optimal solution of the model in both settings: $\mu=\infty$ and $\mu<\infty$. In Section $\mathrm{V}$ we compute the steady-state job-length distribution, which enables us in Section VI to characterize the optimal threshold policy. Finally, Section VII illustrates the obtained solution and its features through different numerical examples.

\section{RELATED WORK}

Scheduling multi-cast traffic with deadlines has various applications, e.g., wireless sensor networks and video streams over cellular networks [4]. Considerable attention has been given in the literature to systems where the specific deadlines of requests are known when the requests are made. This gives rise to well studied scheduling problems for queues and networks of queues with deadline-aware scheduling disciplines like Earliest Deadline First (EDF). For example, earliest deadline first queues are investigated under heavy traffic conditions in [5] and [6]. The optimality of EDF in terms of numbers of jobs that meet their deadline was shown in [7], assuming exponentially distributed service requirements. EDF and related schedulers assume that there is a separate "service" for each job, whereas in our setting similar requests can be bundled. This opens up a whole new dimension of research.

Although the mechanics are completely different, at a higher level of abstraction, the features of waiting for similar requests and sharing the same transmission exhibits similarities with the operation of batch-service queues [8] and gated service disciplines [9].

The models that we come across show interesting resemblances with a wide variety of models from very different application fields, including the shot-noise process (see, for example, [10]) and server routing in polling systems [11].

\section{MODEL DESCRIPTION}

We consider an $\mathrm{M} / \mathrm{M} / 1$ queue with batch service, infinite service capacity and job abandonment. Jobs arrive to the queue according to a Poisson process with rate $\lambda$ and have an exponentially distributed service requirement with mean $1 / \mu$, which is independent of the batch size. Jobs that are waiting in the queue abandon after an exponentially distributed amount of time with mean $1 / \theta$. Furthermore, all interarrival times, service requirements and abandonment times are independent.

At each time the policy $\phi$ chooses whether to process the jobs waiting in the queue or not. Once a job has been admitted for service we assume that it can not abandon the system. Let $N^{\phi}(t) \in\{0,1, \ldots\}$ denote the number of jobs waiting in the queue at time $t$ under the policy $\phi$. Let $S^{\phi}\left(N^{\phi}(t)\right) \in\{0,1\}$ denote the decision at time $t$ under policy $\phi$ when there are
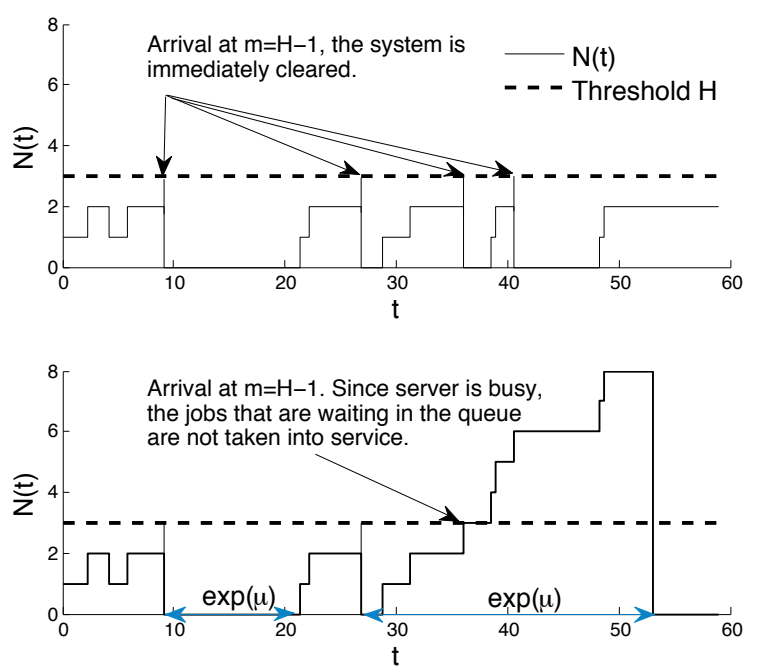

Fig. 1: Simulation of process $N(t)$, number of waiting jobs at time $t$, under threshold $H=3$. Above the $\mu=\infty$ case, below the $\mu<\infty$ case. Below $\exp (\mu)$ refers to the busy period of the server, which is exponentially distributed with rate $\mu<\infty$. As a consequence $N(t)$ not only depends on $H$ but also on the length of each busy period.

$N^{\phi}(t)$ jobs present in the queue. Namely, $S^{\phi}\left(N^{\phi}(t)\right)=0$ if the server idles, and $S^{\phi}\left(N^{\phi}(t)\right)=1$ if the server decides to take a batch into service. Due to the infinite capacity of the server we assume that, as soon as the server is activated, i.e., $S^{\phi}\left(N^{\phi}\right)(t)=1$, all jobs that are waiting in the queue initialize their service. Hence, the batch size upon activation equals the number of jobs waiting in the queue, $N^{\phi}(t)$.

We will analyze this problem in two different settings (see Figure 1):

- $\quad$ The system is cleared as soon as the decision of taking a batch into service is made, that is, $\mu=\infty$.

- The service speed is limited and is exponentially distributed with rate $\mu<\infty$.

In the first case $(\mu=\infty)$, since service is immediate, the server empties the system as soon as the decision of activating the service is made. This means that whenever the policy $\phi$ decides to activate service, a batch of size $N^{\phi}(t)$ (i.e., all jobs waiting in the queue) will be instantaneously processed. In the second case $(\mu<\infty)$ upon activation, the server takes a batch of size $N^{\phi}(t)$ into service, and allocates an exponentially distributed amount of time to process it. While the server is busy, new jobs might arrive to the queue. In this case, the server is not allowed to take a new batch into service until service completion of the previous batch; see Figure 1 (below) around $t=37$. Hence, the evolution of $N^{\phi}(t)$ depends on both the policy $\phi$ and the state of the server (i.e., idling or busy). In the $\mu=\infty$ case, the state of the system reduces to the number of jobs waiting in the queue. In the $\mu<\infty$ case, the state of the system is given by $(m, a)$, where $m$ denotes the number of jobs waiting in the queue and $a \in\{0,1\}$ indicates whether the server is busy $(a=1)$ or idles $(a=0)$.

Let us denote by $C_{h}$ the cost per unit time of holding jobs in the queue, by $C_{a}$ the penalty for jobs abandoning the queue, 
and by $C_{s}$ the set-up cost. The latter is incurred every time the server is activated in the case $\mu=\infty$ and incurred per unit of time the server is active in the case $\mu<\infty$. The objective of the present work is to find the policy $\phi$ so as to minimize

$\limsup _{T \rightarrow \infty} \frac{1}{T} \mathbb{E}\left[\int_{0}^{T}\left[C_{h} N^{\phi}(t)+C_{s} S^{\phi}\left(N^{\phi}(t)\right)\right] \mathrm{d} t+C_{a} R^{\phi}(T)\right]$,

where $R^{\phi}(T)$ denotes the number of jobs that abandon in the interval $[0, T]$. By Dynkin's formula [12], we have $C_{a} \mathbb{E}\left[R^{\phi}(t)\right]=C_{a} \theta \mathbb{E}\left[N^{\phi}(t)\right]$, and therefore the latter objective function is equivalent to finding $\phi$ that minimizes

$$
\limsup _{T \rightarrow \infty} \frac{1}{T} \mathbb{E}\left[\int_{0}^{T}\left[\tilde{C} N^{\phi}(t)+C_{s} S^{\phi}\left(N^{\phi}(t)\right)\right] \mathrm{d} t\right],
$$

where $\tilde{C}=C_{h}+\theta C_{a}$. Due to ergodicity of the system, the time-average optimal policy is equivalent to the averaged-overspace optimal policy, and hence we want to find $\phi$ such that

$$
\min _{\phi}\left(\tilde{C} \mathbb{E}\left[N^{\phi}\right]+C_{s} P_{b}^{\phi}\right)
$$

In this last equation $P_{b}^{\phi}$ stands for the probability that the setup cost is paid under policy $\phi$, i.e., $P_{b}^{\phi}=\mathbb{P}\left(S^{\phi}\left(N^{\phi}\right)=1\right)$. The problem described above is a Markov Decision Process and we refer to it as Problem $\mathrm{P}$ throughout the paper.

In general these types of problems are very complicated to solve due to the curse of dimensionality and the unbounded transition rates which yield a non-uniformizable system. However, we will observe in Section IV that the result in [2] allows structural results to be proven for Problem P. We will show that in both settings, $\mu=\infty$ and $\mu<\infty$, monotone policies are average optimal. That is, there exists a threshold $H$ for which the system prescribes not to take jobs into service for all states $m \leq H-1$, and serves them otherwise.

\section{OPTIMALITY OF THRESHOLD POLICIES}

The optimality of monotone policies has been broadly studied in the literature on uniformizable problems; see, e.g., [13], [14]. However, obtaining structural results for nonuniformizable problems is much more involved. In a recent study, the authors in [2] have developed a methodology to overcome the problem originated by unbounded jump Markov processes. This method is known as the Smoothed Rate Truncation method (SRT) and consists of approximating the infinite state Markov Decision Process (MDP) by finite state MDPs. The relative value function that corresponds to the original MDP can then be obtained as a limit of the relative value functions of the finite state MDPs. Moreover, the limiting value function keeps the same structural properties, namely convexity and supermodularity. In this section we start by establishing that the requirements to apply the SRT are satisfied herein. Later, in Proposition 1, we prove that monotone policies are optimal for Problem P.

Let us first define the finite state MDP, that is, let $L<\infty$ and let the number of jobs waiting in the queue be $m \in$ $\{0, \ldots, L\}$. Furthermore, let us smooth the arrival rate as follows: $q^{\phi, L}(m-1, m)=\lambda\left(1-\frac{m-1}{L}\right)$, for all $L \geq m \geq 1$. This truncation now yields bounded transition rates, and the smoothing of the arrival rate guarantees the structure of the original value function to be maintained. We refer to this finite state space MDP as Problem T.

In Lemma 1, we show that the assumptions in [2, Theorem 3.1] hold for Problem P, making SRT suitable for the model under study; the proof can be found in [15]. We first introduce the following definition which will be needed in Lemma 1.

Definition 1 Let $E_{r}$ be an increasing sequence as $r \rightarrow \infty$ such that $E_{r} \rightarrow E$, where $E$ is the state space. Further let $h: E \rightarrow \mathbb{R}_{+}$be such that $\inf \left\{h(m): m \notin E_{r}\right\} \rightarrow \infty$ as $r \rightarrow \infty$. Then $h$ is called a moment function.

Lemma 1 Let $q^{\phi, L}(m, \tilde{m})$ denote the transition rate from state $m$ to state $\tilde{m}$ under policy $\phi$ and truncation parameter L. Then Problem $T$ satisfies the following conditions.

- $\quad$ There exists a function $h: \mathbb{N} \cup\{0\} \rightarrow \mathbb{R}_{+}$, constants $k_{1}, k_{2}>0$ and $M>0$ such that for all $\phi$ and $L$

$$
\sum_{\tilde{m}=0}^{\infty} q^{\phi, L}(m, \tilde{m}) h(\tilde{m}) \leq-k_{1} h(m)+k_{2} \mathbb{1}_{\{m<M\}}(m) .
$$

- $\quad$ The functions $(\phi, L) \rightarrow q^{\phi, L}(m, \tilde{m})$ and $(\phi, L) \rightarrow$ $\sum_{\tilde{m}} q^{\phi, L}(m, \tilde{m}) h(\tilde{m})$ are continuous in $\phi$ and $L$.

Having proven that Problem $\mathrm{T}$ satisfies the conditions in Lemma 1, we next prove that monotone policies, which in our setting reduce to threshold policies, are optimal for Problem P. A sketch of the proof can be found below for the case $\mu=\infty$ and found in Appendix IX-A for the case $\mu<\infty$.

Proposition 1 Letting $\mu \in \mathbb{R}_{+} \cup\{\infty\}$, then there exists a threshold policy $H$ such that it is optimal for Problem $P$.

Proof: Consider the case $\mu=\infty$ (refer to the appendix for the case $\mu<\infty)$. Let us denote by $V^{\infty}(m)$ the value function corresponding to Problem P, and let $g^{\infty}$ be the average cost incurred by an optimal policy. Recall that the state of the system in this framework reduces to $m$, the number of jobs waiting in the queue. Then, $V^{\infty}(m)$ satisfies the Bellman equation [13] for all $m \geq 0$

$$
\begin{aligned}
& (\lambda+\theta m) V^{\infty}(m)+g^{\infty}=\tilde{C} m+\min \left\{\lambda V^{\infty}(m+1)\right. \\
& \left.+\theta m V^{\infty}\left((m-1)^{+}\right), C_{s}+(\lambda+\theta m) V^{\infty}(0)\right\} .
\end{aligned}
$$

Proving that a threshold policy is optimal is equivalent to proving that passive action being optimal in state $m+1 \geq 1$ implies passive action to be optimal in state $m \geq 0$. Namely,

$$
\begin{aligned}
& \lambda V^{\infty}(m+2)+\theta(m+1) V^{\infty}(m) \\
& \leq C_{s}+(\lambda+\theta(m+1)) V^{\infty}(0)
\end{aligned}
$$

implies

$$
\lambda V^{\infty}(m+1)+\theta m V^{\infty}(m-1) \leq C_{s}+(\lambda+\theta m) V^{\infty}(0) .
$$

It is easy to see that $V^{\infty}(\cdot)$ being a non-decreasing function is a sufficient condition for the latter implication to be satisfied. Since $V^{\infty}(\cdot)$ being non-decreasing implies $\lambda\left(V^{\infty}(m+1)-\right.$ $\left.V^{\infty}(0)\right)+\theta m\left(V^{\infty}(m-1)-V^{\infty}(0)\right) \leq \lambda\left(V^{\infty}(m+2)-\right.$ $\left.V^{\infty}(0)\right)+\theta(m+1)\left(V^{\infty}(m)-V^{\infty}(0)\right) \leq C_{s}$. 
Now recall that Problem $\mathrm{P}$ is non-uniformizable and therefore we use the SRT approach. To apply SRT we truncate the system with the parameter $L$, and define smoothed arrival transition rates $q(m, m+1)=\lambda\left(1-\frac{m}{L}\right)$ for all $0 \leq m \leq L$. We denote by $V^{\infty, L}(\cdot)$ the value function of the truncated system. By Lemma 1 and [2, Theorem 3.1] we have that $V^{\infty, L} \rightarrow V^{\infty}$ as $L \rightarrow \infty$ component wise. Therefore, it suffices to prove that $V^{\infty, L}$ is a non-decreasing function, as this implies $V^{\infty}$ to be non-decreasing. The proof of $V^{\infty, L}(m)$ being non-decreasing can be found in [15].

We have proven threshold policies to be optimal for Problem P. This allows us to study the stationary behavior of the system under this type of policies.

\section{STEADY-STATE DISTRIBUTION}

In this section we derive the steady-state distribution of Problem $\mathrm{P}$ under policies of threshold type. We will refer to these policies as threshold $H$ or simply $\phi=H$, when the threshold is determined by $H \in\{0,1, \ldots\}$. Recall that threshold $H$ prescribes to be passive for all states $m \leq H-1$, and active for all $m \geq H$. Throughout this section, for clarity of exposition, we drop the dependency on $H$ from the notation.

In $\mathrm{V}$-A we derive the steady-state distribution of Problem $\mathrm{P}$ for the case $\mu=\infty$ and in V-B for the case $\mu<\infty$.

\section{A. Steady-state distribution in the case $\mu=\infty$}

In the case of infinite service speed we assume that the system empties the queue as soon as the decision to serve is made. In this setting, under threshold $H$, the state space is given by $E=\{0,1, \ldots, H-1\}$. As soon as an arrival occurs in state $m=H-1$ the system is immediately cleared.

Let us define $\pi_{m}$ as the steady-state probability of being in state $m \in\{0, \ldots, H-1\}$. Hence, we have the following balance equations

$$
\lambda \pi_{m-1}=\theta m \pi_{m}+\lambda \pi_{H-1}, \quad \forall 0<m \leq H-1,
$$

together with the normalizing equation $\sum_{i=0}^{H-1} \pi_{i}=1$.

Solving these balance equations results in

$$
\pi_{m}=\pi_{H-1}\left(1+\sum_{i=1}^{H-1-m}\left(\frac{\theta}{\lambda}\right)^{i} \frac{(m+i) !}{m !}\right),
$$

for all $m=0,1, \ldots, H-1$. Calculations can be found in [15]. Moreover, from the normalization equation, we obtain

$$
\pi_{H-1}=\left(\sum_{m=0}^{H-1}\left(1+\sum_{i=1}^{H-1-m}\left(\frac{\theta}{\lambda}\right)^{i} \frac{(i+m) !}{m !}\right)\right)^{-1}
$$

We therefore have obtained the expression for all $\pi_{m}$ and $0 \leq$ $m \leq H-1$.

\section{B. Steady-state distribution in the case $\mu<\infty$}

In the case of finite service speed, the state of the system is given by $(m, a)$ where $m$ denotes the number of jobs waiting in the queue and $a \in\{0,1\}$ whether the server is available $(a=0)$ or busy $(a=1)$. Observe that under threshold $\mathrm{H}$, during the idle period of the server, the number of jobs in the queue, i.e., $m$, takes values in the set $\{0, \ldots, H-1\}$. The latter means that, as soon as an arrival happens in state $m=H-1$, the server activates the service with a batch of $H$ jobs. Once the server is active, during the time the batch is being processed (exponentially distributed with mean $1 / \mu$ ), the number of jobs waiting in the queue are such that $m \in \mathbb{N} \cup\{0\}$. That is, even if the threshold $H$ is reached, the activation of the server is postponed until it completes processing the previous batch.

Let us denote by $\pi(m, a)$ the steady-state probability of being in state $(m, a)$, for all $m \geq 0$ and $a \in\{0,1\}$, and assume $\pi(m, 0)=0$ for all $m \geq H$. As in the previous section, we omit the dependence on $H$ from the notation. Then, $\pi(m, a)$ for all $m \in \mathbb{N} \cup\{0\}$ and $a \in\{0,1\}$ can be derived from the following balance equations: for all $m \in \mathbb{N}$

$$
\begin{aligned}
& (\lambda+m \theta+\mu) \pi(m, 1) \\
& =\lambda \pi(m-1,1)+(m+1) \theta \pi(m+1,1),
\end{aligned}
$$

and for all $0 \leq m \leq H-1$

$$
\begin{aligned}
& (\lambda+m \theta) \pi(m, 0) \\
& =\lambda \pi(m-1,0)+\mu \pi(m, 1)+(m+1) \theta \pi(m+1,0) .
\end{aligned}
$$

In order to solve the balance equations in (2) and (3) we will use their corresponding ordinary generating functions. Observe in Equation (3) that the steady-state probabilities of the idle period depend on the steady-state probabilities of the busy period. Therefore, we first obtain the closed-form expression of $\pi(m, 1)$ for all $m$, and using these expressions we derive those that correspond to the idle period, i.e., $\pi(m, 0)$ for all $H-1 \geq m \geq 0$. The explicit expression of the probabilities are presented in Propositions 2. The calculations to derive these expressions can be found in Appendix IX-B1, for the busy period, and in Appendix IX-B2, for the idle period.

Proposition 2 Let $\pi(m, 1)=a_{1}(m) \pi(0,1)$ where $a_{1}(0):=1$,

$$
\begin{aligned}
a_{1}(1):= & \frac{\lambda+\mu}{\theta}-\frac{\mathrm{e}^{\lambda / \theta}}{\sum_{i=0}^{\infty} \frac{(\lambda / \theta)^{i}}{i !(\mu / \theta+i)},} \\
a_{1}(m):= & \frac{1}{m !} \sum_{k=0}^{m}\left(\begin{array}{c}
m \\
k
\end{array}\right)\left(\frac{\sum_{j=0}^{\infty} \frac{\left(\frac{\lambda}{\theta}\right)^{j} \ell_{k}\left(-\frac{\mu}{\theta}-j\right)}{j !\left(\frac{\mu}{\theta}+j\right)}}{\sum_{i=0}^{\infty} \frac{\left(\frac{\lambda}{\theta}\right)^{i}}{i !\left(\frac{\mu}{\theta}+i\right)}}\right. \\
& \left.\cdot \sum_{i=0}^{m-k}\left(\begin{array}{c}
m-k \\
i
\end{array}\right)\left(\frac{\lambda}{\theta}\right)^{m-k-i} \ell_{i}\left(\frac{\mu}{\theta}\right)\right),
\end{aligned}
$$

for all $m \geq 2$, and $\ell_{k}(x)$ the Pochhammer symbol. Let $\pi(m, 0)=a_{0}^{H}(m) \pi(0,1)$ where $a_{0}^{H}(H-1):=$ $\frac{\mu}{\lambda} \sum_{m=0}^{H-1} a_{1}(m)$

$$
\begin{aligned}
a_{0}^{H}(m):= & \left.\frac{\mu}{\lambda} \sum_{r=0}^{H-1} a_{1}(r)\right) \sum_{i=0}^{H-1-m}\left(\frac{\theta}{\lambda}\right)^{i} \frac{(m+i) !}{m !} \\
& -\frac{\mu}{\lambda} \sum_{r=m+1}^{H-1} a_{1}(r)\left(\sum_{i=0}^{r-m-1}\left(\frac{\theta}{\lambda}\right)^{i} \frac{(m+i) !}{m !}\right),
\end{aligned}
$$

for all $H-2 \geq m>0$, and $a_{0}^{H}(0):=\frac{\mu}{\lambda}+\frac{\theta}{\lambda} a_{0}^{H}(1)$. If $\pi(0,1)$ is such that $\sum_{m=0}^{H-1} \pi(m, 0)+\sum_{m=0}^{\infty} \pi(m, 1)=1$, that is, if

$$
\pi(0,1)=\left(\sum_{m=0}^{H-1} a_{0}^{H}(m)+\sum_{m=0}^{\infty} a_{1}(m)\right)^{-1}
$$


then $\pi(m, 1)$ and $\pi(m, 0)$ solve Equations (2) and (3).

Once we have obtained all $\pi(m, 0)$ for all $H-1 \geq m \geq 0$, and $\pi(m, 1)$ for all $m \geq 0$ we can proceed to compute the mean number of jobs in the system under threshold policy $H$, as well as the mean amount of time the set-up cost is incurred. In the next section we show that this provides a characterization of the optimal threshold.

\section{CHARACTERIZATION OF THE OPTIMAL THRESHOLD}

In this section we characterize the optimal threshold policy using the steady-state probabilities that we have computed above. This characterization, as we will see, depends on the set-up cost $C_{s}$. For the infinite service speed case $(\mu=\infty)$, the characterization of the optimal threshold is explicit whose solution we present in Proposition 3. In the finite service speed case, the characterization of the optimal threshold is determined by a function that is subject to optimization; see Proposition 4.

From Proposition 1 we know that threshold policies are optimal for Problem P. Then Problem P can be rewritten as

$$
\min \left(\tilde{C} \mathbb{E}\left(N^{H}\right)+C_{s} P_{b}^{H}\right),
$$

recalling $P_{b}^{H}=P\left(S^{H}\left(N^{H}\right)=1\right)$. The properties of $P_{b}^{H}$ will play an important role in the characterization of the optimal $H$. Therefore, in the following definition we provide the expression of $P_{b}^{H}$ according to the value of $\mu$.

Definition 2 We denote by $P_{b}^{H}$ the probability that the set-up $\operatorname{cost} C_{s}$ is incurred under threshold policy $H$, i.e.:

- In the $\mu=\infty$ case, it is the probability of being in state $H-1$ with the next event being an arrival. Namely, $P_{b}^{H}:=\pi_{H-1}^{H} \frac{\lambda}{\lambda+\theta(H-1)}$ where $\pi_{H-1}^{H}$ is given by (1) after adding the superscript $H$.

- In the $\mu<\infty$ case, it is the probability that the server is busy. Namely, $P_{b}^{H}:=\sum_{m=0}^{\infty} \pi^{H}(m, 1)$ where $\pi^{H}(m, 1)$ for all $m \geq 0$ are given by Proposition 2 after adding the superscript $H$.

In the following proposition we propose an explicit representation of the optimal threshold.

Proposition 3 Let us define $\alpha(H)$ such that

$$
\alpha(H):=\tilde{C} \frac{\mathbb{E}\left(N^{H}\right)-\mathbb{E}\left(N^{H-1}\right)}{P_{b}^{H-1}-P_{b}^{H}} .
$$

If $\alpha(H)$ is non-decreasing in $H, P_{b}^{H}$ is non-increasing in $H$, and if $\alpha(H) \leq C_{s}<\alpha(H+1)$, then $H$ is optimal for Problem $P$.

Proof: We present here a sketch of the proof. We aim at proving that for all $H^{\prime} \neq H, \tilde{C} \mathbb{E}\left(N^{H}\right)+C_{s} P_{b}^{H} \leq \tilde{C} \mathbb{E}\left(N^{H^{\prime}}\right)+$ $C_{s} P_{b}^{H^{\prime}}$. We assume $H^{\prime}<H$, and the opposite can be handled similarly. By assumption we have $\alpha(H-1) \leq \alpha(H)$, for all $H \geq 1$, and then using simple algebra one can obtain

$$
\alpha(H-1) \leq \tilde{C} \frac{\mathbb{E}\left(N^{H}\right)-\mathbb{E}\left(N^{H-2}\right)}{P_{b}^{H-2}-P_{b}^{H}} \leq \alpha(H) \leq C_{s},
$$

We make the following induction assumption for $a>0$

$$
\alpha(H-a+1) \leq \tilde{C} \frac{\mathbb{E}\left(N^{H}\right)-\mathbb{E}\left(N^{H-a}\right)}{P_{b}^{H-a}-P_{b}^{H}} \leq C_{s} .
$$

By assumption we have $\alpha(H-a) \leq \alpha(H-a+1)$, and hence, using simple calculations, we obtain from (4)

$$
\tilde{C} \frac{\mathbb{E}\left(N^{H}\right)-\mathbb{E}\left(N^{H-a-1}\right)}{P_{b}^{H-a-1}-P_{b}^{H}} \leq \tilde{C} \frac{\mathbb{E}\left(N^{H}\right)-\mathbb{E}\left(N^{H-a}\right)}{P_{b}^{H-a}-P_{b}^{H}} \leq C_{s} .
$$

From (5) and some algebra we deduce that $\alpha(H-a) \leq$ $\tilde{C} \frac{\mathbb{E}\left(N^{H}\right)-\mathbb{E}\left(N^{H-a-1}\right)}{P^{H-a-1}-P_{b}^{H}}$. This together with (5) yields $\alpha(H-a) \leq$ $\tilde{C} \frac{\mathbb{E}\left(N^{H}\right)-\mathbb{E}\left(N^{H}-a-1\right.}{P_{b}^{H-a-1}-P_{b}^{H}} \leq C_{s}$, thus concluding the induction. For all $0<a \leq H-1$ denote $H^{\prime}=H-a$. We have then proven that for all $H^{\prime}<H$

$$
\begin{aligned}
& \tilde{C} \frac{\mathbb{E}\left(N^{H}\right)-\mathbb{E}\left(N^{H^{\prime}}\right)}{P_{b}^{H^{\prime}}-P_{b}^{H}} \leq C_{s} \\
& \Longrightarrow \tilde{C} \mathbb{E}\left(N^{H}\right)+C_{s} P_{b}^{H} \leq \tilde{C} \mathbb{E}\left(N^{H^{\prime}}\right)+C_{s} P_{b}^{H^{\prime}},
\end{aligned}
$$

which concludes the proof.

In the following lemma we prove that the assumptions in Proposition 3 hold in the case $\mu=\infty$. The proof can be found in [15].

Lemma 2 Let $\mu=\infty$, and $\pi_{m}^{H}$ as given in Section $V$-A after adding the superscript $H$. Then

- $P_{b}^{H}=\pi_{H-1}^{H} \lambda /(\lambda+\theta(H-1))$, the probability at which the set-up cost $C_{s}$ is paid, is convex non-increasing.

- The function $\alpha(H)$, as defined in Proposition 3, is non-decreasing.

Corollary 1 Assume $\mu=\infty$ and define $\alpha(1):=-\infty$. Then, if $\alpha(H) \leq C_{s}<\alpha(H+1)$ for all $H \geq 1, H$ is the optimal threshold policy for Problem $P$.

Proof: The proof follows from Proposition 3 and Lemma 2.

In the case $\mu<\infty$ we could not prove $\alpha$ to be nondecreasing. The optimal threshold then has to be characterized differently. This characterization is given in the following proposition.

Proposition 4 Let $\mathcal{N}_{i}=\mathbb{N} \backslash\left\{0, \ldots, H_{i}\right\}$ for a given $H_{i}$, let $P_{b}^{H}$ be non-increasing, and define $\beta(\cdot)$ as follows:

Step i. Compute

$$
\beta\left(H_{i}\right):=\inf _{H \in \mathcal{N}_{i-1}} \frac{\mathbb{E}\left(N^{H}\right)-\mathbb{E}\left(N^{H_{i-1}}\right)}{P_{b}^{H_{i-1}}-P_{b}^{H}}, i \geq 1,
$$

and denote by $H_{i}$ the largest $H \in \mathcal{N}_{i-1}$ such that (6) is minimized. If $H_{i}=\infty$ stop, otherwise jump to step $i+1$.

Then, $\beta\left(H_{i}\right)$ is non-decreasing in $H_{i}$ and if $\beta\left(H_{i}\right) \leq C_{s}<$ $\beta\left(H_{i}+1\right)$, then $H_{i}$ is optimal for Problem P. Moreover, if $C_{s}<\beta\left(H_{1}\right)$, then it will be optimal to always serve. 
TABLE I: Example 1: Minimum set-up cost $C_{s}$ such that $H$ is optimal.

\begin{tabular}{c|c|c|c|c|c|c}
\hline$H$ & 1 & 2 & 3 & 4 & 5 & 6 \\
\hline$C_{s}$ & $-\infty$ & 0.6096 & 2.4359 & 6.2595 & 12.8192 & 22.5343 \\
\hline
\end{tabular}

Proof: Let us first prove that $\beta\left(H_{i}\right)$ is non-decreasing in $H_{i}$. Recall that by definition

$$
\frac{\mathbb{E}\left(N^{H_{i}}\right)-\mathbb{E}\left(N^{H_{i-1}}\right)}{P_{b}^{H_{i-1}}-P_{b}^{H_{i}}} \leq \frac{\mathbb{E}\left(N^{H_{i+1}}\right)-\mathbb{E}\left(N^{H_{i-1}}\right)}{P_{b}^{H_{i-1}}-P_{b}^{H_{i+1}}},
$$

and then $\left(\mathbb{E}\left(N^{H_{i}}\right)-\mathbb{E}\left(N^{H_{i-1}}\right)\right)\left(P_{b}^{H_{i-1}}-P_{b}^{H_{i+1}}\right) \leq$ $\left.\left(\mathbb{E}\left(N^{H_{i+1}}\right)-\mathbb{E}\left(N^{H_{i-1}}\right)\right)\right)\left(P_{b}^{H_{i-1}}-P_{b}^{H_{i}}\right)$. Upon adding and subtracting $\mathbb{E}\left(N^{H_{i}}\right)\left(P_{b}^{H_{i-1}}-P_{b}^{H_{i}}\right)$ on the RHS, and after some algebra, we obtain $\beta\left(H_{i}\right) \leq \beta\left(H_{i}+1\right)$.

Having proven that $\beta(\cdot)$ is non-decreasing, the optimality of threshold $H_{i}$ if $\beta\left(H_{i}\right) \leq C_{s}<\beta\left(H_{i}+1\right)$ can be proven in the same way as in Proposition 3.

The following lemma establishes the characterization proposed in Proposition 4 to hold when $\mu<\infty$. The proof can be found in [15].

Lemma 3 Assume $\mu<\infty$ and $\pi^{H}(m, a)$ as given in Section $V$-B, for all $m \geq 0$ and $a \in\{0,1\}$, after adding the superscript $H$. Then, $P_{b}^{H}=\pi^{H}(0,1) \sum_{m=0}^{\infty} a_{1}(m)$, the probability of being active, is non-increasing.

We characterize the optimal solution by $\beta\left(H_{i}\right)$ as defined in Proposition 4 in the following corollary for the case $\mu<\infty$.

Corollary 2 Assume $\mu<\infty$ and $\beta\left(H_{i}\right) \leq C_{s}<\beta\left(H_{i}+1\right)$ with $\beta$ defined as in Proposition 4 , then $H_{i}$ is the optimal threshold policy for Problem P. And if $C_{s}<\beta\left(H_{1}\right)$ then 0 is the optimal threshold (always serve).

Proof: The proof follows from Proposition 4 and Lemma 3.

In the next section we analyze the optimal threshold policies in both frameworks.

\section{EXAMPLES}

In this section we illustrate the features of the optimal threshold policies that have been characterized in Section VI through different examples. In Examples 1 and 2 we illustrate the optimal threshold policy for different values of the setup cost $C_{s}$ and we evaluate the performance of non-optimal threshold policies in comparison with the optimal one for the case $\mu=\infty$ and $\mu<\infty$, respectively. In Examples 3 and 4 we consider the influence on the optimal threshold policy for varying values of $\theta$ in the case $\mu=\infty$ and for varying values of $\theta$ and $\mu$ in the case $\mu<\infty$.

Example 1: Let us assume $\lambda=4, \mu=\infty, \theta=1.5$ and $\tilde{C}=1$. Then the minimum value of $C_{s}$ such that $H$ is optimal for Problem $\mathrm{P}$ is given by $\alpha(H)$, whose values are presented in Table I. In Figure 2 (left) we illustrate this optimal solution, where we plot the average cost $\mathbb{E}\left(N^{H}\right)+C_{s} P_{b}^{H}$ incurred by
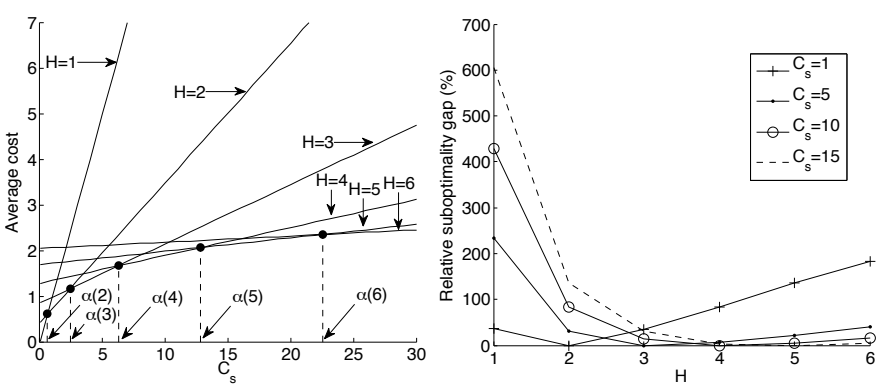

Fig. 2: On the left the average cost under different threshold policies and varying value of $C_{s}$. On the right the relative suboptimality gap of different threshold policies with respect to the optimal threshold.

TABLE II: Example 2: Minimum set-up cost $C_{s}$ such that $H$ is optimal.

\begin{tabular}{c|c|c|c|c|c|c}
\hline$H$ & 0 & 1 & 2 & 3 & 4 & 5 \\
\hline$C_{s}$ & $-\infty$ & -2 & -1.151 & -0.2581 & 0.7157 & 1.7937 \\
\hline
\end{tabular}

policy $H$, for different values of $C_{s}$. We only present the solution up to $H=6$, noting that a characterization for all $H$ can be found. In Figure 2 (right) we present the relative sub optimality gap of non-optimal threshold policies with respect to the optimal that we have just characterized. Observe that their performance is very poor.

Example 2: Let us assume $\lambda=2, \mu=0.5, \theta=0.5$ and $\tilde{C}=1$. Then the minimum value of $C_{s}$ such that $H$ is optimal for Problem $\mathbf{P}$ is given by $\beta\left(H_{i}\right)$ which coincides with $\alpha(i)$ in this case. The values of $\beta\left(H_{i}\right)$ are presented in Table II for $H_{i}=i$ up to 5 . We observe that under the assumption $C_{s}>0$ thresholds $H=0,1,2$ are never optimal in this particular example, which means that when there are 1 or 2 jobs waiting to be served the server will idle. These results are illustrated in Figure 3 (left), where we plot the average cost $\mathbb{E}\left(N^{H}\right)+C_{s} P_{b}^{H}$ incurred by policy $H$ for different values of $C_{s}$. In Figure 3 (right) we plot the relative sub optimality gap of different threshold policies with respect to the optimal threshold, and observe that non-optimal thresholds incur a huge cost.
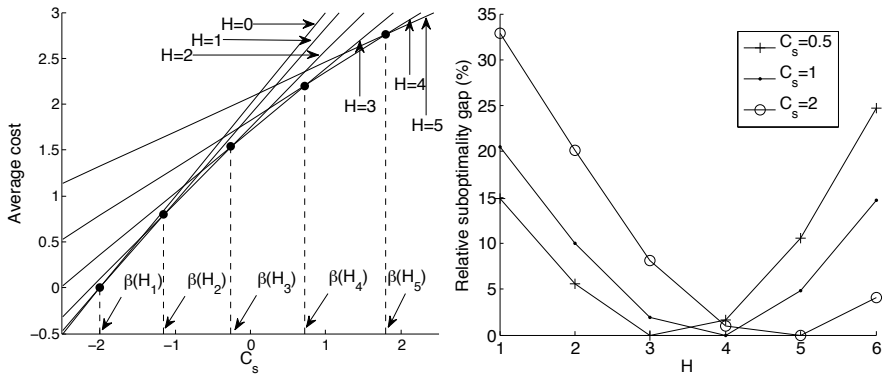

Fig. 3: On the left the average cost under different threshold policies and varying value of $C_{s}$. On the right the relative suboptimality gap of different threshold policies with respect to the optimal threshold. 

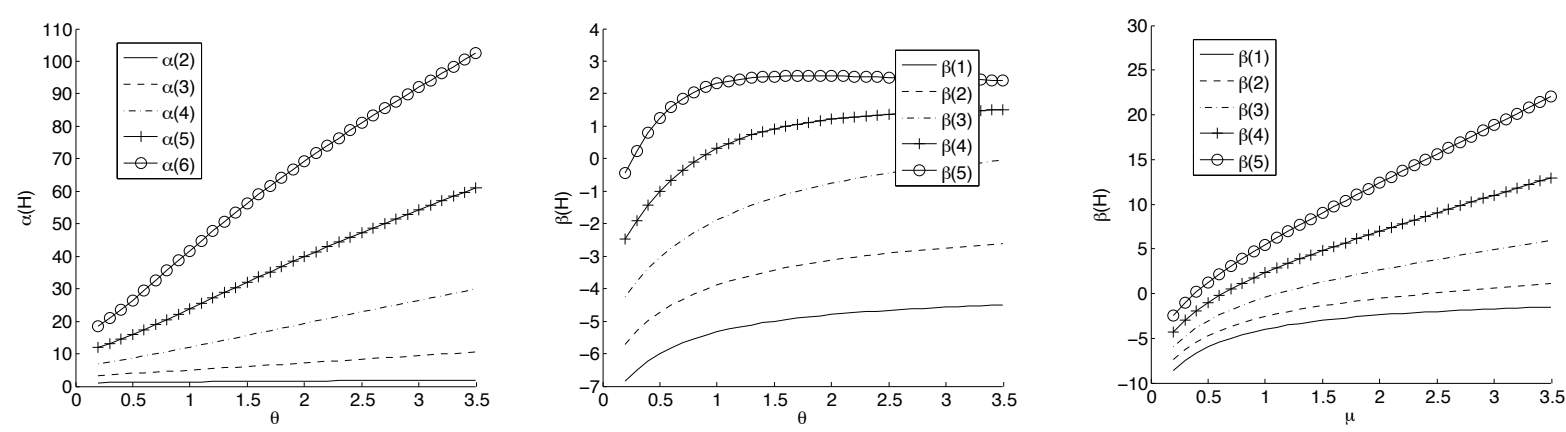

Fig. 4: We plot the values of $\alpha$ and $\beta$ for varying parameters $\theta$ and $\mu$. On the left the case $\mu=\infty$ for varying values of $\theta$. In the middle and on the right the case $\mu<\infty$ for varying values of $\theta$ and $\mu$ respectively.

In the following two examples we observe the policy changes as $\theta$ and $\mu$ vary.

Example 3: Let us assume $\lambda=4, \mu=\infty$ and $C_{h}=C_{a}=1$ and let $\theta$ vary in the interval $[0,3.5]$. We observe in Figure 4 (left) that the minimum value of $C_{s}$ such that threshold policy $H$ is optimal increases as $\theta$ increases. This means that for a fixed set-up cost and for increasing abandonment rates, the system prescribes to activate service earlier to avoid incurring the abandonment penalty.

Example 4: Let us assume $\lambda=2$ and $C_{h}=C_{a}=1$. We first consider the case $\mu=0.5$ and let $\theta$ vary in the interval $[0,3.5]$; see Figure 4 (middle). We observe that as $\theta$ grows large, $\beta(H)$ becomes constant. This phenomenon is explained by the fact that abandonments happen both in the busy period and in the idle period of the server, and hence for a fixed set-up cost the threshold is maintained as $\theta$ grows. Secondly, we consider $\theta=0.5$ and let $\mu$ vary in the interval $[0,3.5]$. We observe in Figure 4 (right) that the faster the service, the smaller the optimal threshold for a fixed $C_{s}$.

\section{CONCLUSION}

We have considered a content delivery problem where a single server decides when to multi-cast the content that has been requested. We have obtained an optimal scheduling policy, which turns out to have a very simple structure, and we have characterized it with respect to the set-up cost.

We claim that this characterization is easily implementable and allows future generalizations. For instance, one could consider a multi-class single-server queue where different classes of jobs request a different content. An easy heuristic for this model would be to send the request to the class of jobs with higher positive $\alpha_{k}\left(H^{k}\right)$ or $(\beta(\cdot))$, where $H^{k}$ in this case would represent the number of jobs waiting in the queue $k$.

Finally, one could investigate the fluid limit version of our problem. Different from standard fluid limits, this process still contains randomness due to the random service times that do not disappear in the applied scaling, and hence becomes mathematically very interesting.

\section{REFERENCES}

[1] A. Jean-Marie and E. Hyon, "Scheduling services in a queuing system with impatience and setup costs," Lecture Notes in Electrical Engineering, vol. 62 , pp. $45-50,2010$
[2] S. Bhulai, A. Brooms, and F. Spieksma, "On structural properties of the value function for an unbounded jump Markov process with an application to a processor sharing retrial queue," QUESTA, vol. 76, pp. 425-446, 2014.

[3] M. Larrañaga, U. Ayesta, and I. M. Verloop, "Index policies for a multiclass queue with convex holding cost and abandonment," in Proceedings of ACM SIGMETRICS, 2014.

[4] K. S. Kim, C. Li, and E. Mondiano, "Scheduling multicast traffic with deadlines in wireless networks," Proceedings of IEEE INFOCOM, 2014.

[5] B. Doytchinov, J. Lehoczky, and S. Shreve, "Real-time queues in heavy traffic with earliest-deadline-first queue discipline," The Annals of Applied Probability, vol. 11, no. 2, pp. 332-378, 2001.

[6] L. Kruk, J. Lehoczky, K. Ramanan, and S. Shreve, "Heavy traffic analysis for edf queues with reneging," The Annals of Applied Probability, vol. 21, no. 2, pp. 484-545, 2011.

[7] D. Towsley and S. Panwar, "On the optimality of minimum laxity and earliest deadline first scheduling for real-time multiprocessors," Proceedings of IEEE EUROMICRO-90 Real Time Workshop, pp. 17-24, 1990.

[8] R. Deb and R. Serfozo, "Optimal control of batch service queues," Advances in Applied Probability, vol. 5, pp. 340-361, 1973.

[9] O. Boxma, A. van Wijk, and I. Adan, "Polling systems with a gated/exhaustive discipline," Proc. 3rd Int. Conf. Performance Evaluation Methodologies and Tools (ValueTools), 2008.

[10] O. Kella, "On growth-collapse processes with stationary structure and their shot-noise counterparts," Journal of Applied Probability, vol. 46, no. 2, pp. 363-371, 2009.

[11] J. Dorsman, S.Borst, O.Boxma, and M.Vlasiou, "Markovian polling systems with an application to wireless random-access networks," To appear in Performance Evaluation, 2015.

[12] D. Anderson, Introduction to Stochastic Processes with Applications in the Biosciences. Available at www.math.wisc.edu/ anderson/605F13/Notes/StochBio.pdf: University of Wisconsin at Madison, 2013.

[13] M. L. Puterman, Markov Decision Processes: Discrete Stochastic Dynamic Programming. John Wiley \& Sons, 2005.

[14] K. Papadaki and W. Powell, "Exploiting structure in adaptive dynamic programming algorithms for a stochastic batch service problem," European Journal of Operational Research, vol. 142, pp. 108-127, 2002.

[15] M. Larrañaga, O. Boxma, R. Núñez-Queija, and M. Squillante, "Efficient content delivery in the presence of impatient jobs," Available at https://www.dropbox.com/s/7qi3yb36tvxlc3f/ITC27Proofs.pdf?dl=0.

[16] M. Abramowitz and A. Stegun, Handbook of Mathematical Functions, with Formulas, Graphs and Mathematical Tables. Dover Publications, Inc., New York, 1965.

\section{APPENDIX}

\section{A. Proof of Proposition 1: the case $\mu<\infty$}

Let us denote by $V(m, a)$ the value function corresponding to Problem $\mathrm{P}$ in the case $\mu<\infty$, and let $g$ be the average 
cost incurred by an optimal policy. Recall that the state of the system in this framework reduces to $(m, a)$, where $m$ is the number of jobs waiting in the queue and $a \in\{0,1\}$ denotes the state of the server, busy if $a=1$ and available if $a=0$. The value function $V(m, a)$ for all $m \geq 0$ and $a \in\{0,1\}$ satisfies the Bellman equation [13], namely

$$
\begin{aligned}
& (\lambda+\mu+\theta m) V(m, 0)+g \\
& =\tilde{C} m+\min \left\{\lambda V(m+1,0)+\theta m V\left((m-1)^{+}, 0\right)\right. \\
& \left.+\mu V(m, 0), C_{s}+\lambda V(1,1)+\theta m V(0,1)+\mu V(0,0)\right\}
\end{aligned}
$$

and

$$
\begin{aligned}
& (\lambda+\mu+\theta m) V(m, 1)+g \\
& =\tilde{C} m+\lambda V(m+1,1)+m \theta V\left((m-1)^{+}, 1\right)+\mu V(m, 0) .
\end{aligned}
$$

We will now prove that an optimal policy solving the Bellman equation is of threshold type, that is, if active action is optimal in $m$ then active action is optimal in $m^{\prime} \geq m$. In order to do so, let us first define

$$
\begin{aligned}
& f(m, 0):= \tilde{C} m+\lambda V(m+1,0)+\mu V(m, 0) \\
&+\theta m V(m-1,0), \\
& f(m, 1):=\tilde{C} m+C_{s}+\lambda V(1,1)+\theta m V(0,1)+\mu V(0,0),
\end{aligned}
$$

and $\varphi(m)=\min \left(b \in \arg \min _{a \in\{0,1\}} f(m, a)\right)$. Let $m^{\prime} \geq m$ and $a \geq \varphi\left(m^{\prime}\right)$. It then suffices to show that $\varphi\left(m^{\prime}\right) \geq \varphi(m)$. By definition of $\varphi(\cdot)$

$$
f\left(m^{\prime}, \varphi\left(m^{\prime}\right)\right)-f\left(m^{\prime}, a\right) \leq 0 .
$$

Let us now prove that $V(m, 0)$ is supermodular [2], that is, for all $m^{\prime} \geq m$

$$
f\left(m^{\prime}, a\right)+f\left(m, \varphi\left(m^{\prime}\right)\right) \leq f\left(m^{\prime}, \varphi\left(m^{\prime}\right)\right)+f(m, a) .
$$

Assuming first the case $\varphi\left(m^{\prime}\right)=0$ and $a=0$, then (8) is trivially satisfied; similarly (8) is satisfied in the case $\varphi\left(m^{\prime}\right)=1$ and $a=1$. We are left with the case $\varphi\left(m^{\prime}\right)=0$ and $a=1$, for which (8) reduces to $f(m, 0)-f(m, 1) \leq$ $f\left(m^{\prime}, 0\right)-f\left(m^{\prime}, 1\right)$. Since Problem $\mathrm{P}$ for $\mu<\infty$ is nonuniformizable, we use the SRT approach. We truncate the system with the parameter $L$, and define smoothed arrival transition rates, i.e., $q(m, m+1)=\lambda\left(1-\frac{m}{L}\right)$ for all $m \leq L$. We denote by $V^{L}(\cdot, \cdot)$ the value function of the truncated system. By Lemma 1 and [2, Theorem 3.1], we have that $V^{L} \rightarrow V$ as $L \rightarrow \infty$ and the value function maintains its structural properties. Assume, without loss of generality, $\lambda+\mu+\theta L=1$. Then, applying the Value Iteration algorithm [13] to the truncated system, we have

$$
\begin{aligned}
& V_{t+1}^{L}(m, 0)=\tilde{C} m+\min \left\{\lambda\left(1-\frac{m}{L}\right) V_{t}^{L}(m+1,0)\right. \\
& +\left(\lambda \frac{m}{L}+\mu+\theta(L-m)\right) V_{t}^{L}(m, 0)+\theta m V_{t}^{L}(m-1,0), \\
& \left.C_{s}+\lambda V_{t}^{L}(1,1)+\theta L V_{t}^{L}(0,1)+\mu V_{t}^{L}(0,0)\right\}
\end{aligned}
$$

and

$$
\begin{aligned}
& V_{t+1}^{L}(m, 1)=\tilde{C} m+\lambda\left(1-\frac{m}{N}\right) V_{t}^{L}(m+1,1)+\mu V_{t}^{L}(m, 0) \\
& +\left(\lambda \frac{m}{N}+(N-m) \theta\right) V_{t}^{L}(m, 1)+m \theta V_{t}^{L}(m-1,1),
\end{aligned}
$$

since $V_{t+1}^{L}(m, a)-V_{t}^{L}(m, a)=g$. It suffices to prove that $f_{t}^{L}(m, 0)-f_{t}^{L}(m, 1) \leq f_{t}^{L}\left(m^{\prime}, 0\right)-f_{t}^{L}\left(m^{\prime}, 1\right)$, where

$$
\begin{aligned}
f_{t}^{L}(m, 0)= & \lambda\left(1-\frac{m}{L}\right) V_{t}^{L}(m+1,0)+\theta m V_{t}^{L}(m-1,0) \\
& +\left(\lambda \frac{m}{L}+\mu+\theta(L-m)\right) V_{t}^{L}(m, 0),
\end{aligned}
$$

$f_{t}^{L}(m, 1)=\tilde{C} m+C_{s}+\lambda V_{t}^{L}(1,1)+\theta L V_{t}^{L}(0,1)+\mu V_{t}^{L}(0,0)$.

$V^{L}$ is a non-decreasing function and $f_{t}^{L}(m, 0)-f_{t}^{L}(m, 1) \leq$ $f_{t}^{L}\left(m^{\prime}, 0\right)-f_{t}^{L}\left(m^{\prime}, 1\right)$ for all $m^{\prime} \geq m$ and all $t$, the proof of both of which can be found in [15]. By the value iteration argument $f_{t}^{L} \rightarrow f^{L}$ as $t \rightarrow \infty$, and from the SRT we have that $V^{L} \rightarrow V$ and $f^{L} \rightarrow f$ component-wise. Therefore, supermodularity of $V^{L}$ implies supermodularity of $V$. Having proven (7) and (8), and upon combining them, we have that for all $a \geq \varphi\left(m^{\prime}\right)$ and $m^{\prime} \geq m$

$$
\begin{aligned}
f\left(m, \varphi\left(m^{\prime}\right)\right) & \leq f\left(m^{\prime}, \varphi\left(m^{\prime}\right)\right)-f\left(m^{\prime}, a\right)+f(m, a) \\
& \leq f(m, a) .
\end{aligned}
$$

Hence $\varphi(m) \leq \varphi\left(m^{\prime}\right)$, which concludes the proof.

\section{B. Proof of Proposition 2}

First we derive the expression of $\pi(m, 1)$ and subsequently the expression of $\pi(m, 0)$.

1) Steady-state distribution in the busy period: We first define the ordinary generating function that corresponds to $\pi(m, 1)$ for all $m \in \mathbb{N}_{0}$, that is, $\Pi_{1}(z)=\sum_{m=0}^{\infty} z^{m} \pi(m, 1)$, and recall Equation (2) for all $m \in \mathbb{N}$. Then, upon multiplying Equation (2) for state $m$ with $z^{m}$, namely

$$
\begin{aligned}
& z^{m}(\lambda+m \theta+\mu) \pi(m, 1)=z^{m} \lambda \pi(m-1,1) \\
& +z^{m}(m+1) \theta \pi(m+1,1), \quad \forall m \in \mathbb{Z} \backslash\{0\},
\end{aligned}
$$

and summing up the latter for all $m \in\{1,2, \ldots\}$, we obtain

$$
\begin{aligned}
& (\lambda+\mu)\left(\Pi_{1}(z)-\pi(0,1)\right)+\theta z \frac{\mathrm{d}}{\mathrm{d} z} \Pi_{1}(z) \\
& =\lambda z \Pi_{1}(z)+\theta\left(\frac{\mathrm{d}}{\mathrm{d} z} \Pi_{1}(z)-\pi(1,1)\right) .
\end{aligned}
$$

After some algebra the latter reduces to

$\frac{(\lambda(1-z)+\mu) \Pi_{1}(z)}{-\theta(1-z)}+\frac{\mathrm{d} \Pi_{1}(z)}{\mathrm{d} z}=\frac{(\lambda+\mu) \pi(0,1)-\theta \pi(1,1)}{-\theta(1-z)}$.

We now solve this ordinary differential equation. To do so, let us define $\Pi_{1}^{H}(z)=f_{1}(z) g_{1}(z)$ such that

$$
\frac{\frac{\mathrm{d} f_{1}(z)}{\mathrm{d} z}}{f_{1}(z)}=-\frac{\lambda(1-z)+\mu}{-\theta(1-z)} \Rightarrow f_{1}(z)=\mathrm{e}^{\frac{\lambda z}{\theta}}(1-z)^{-\frac{\mu}{\theta}} .
$$

Substituting $\Pi_{1}(z)=f_{1}(z) g_{1}(z)=\frac{\mathrm{e}^{\lambda z / \theta}}{(1-z)^{\mu / \theta}} g_{1}(z)$ in Equation (10) and dividing both sides of the equality by $-\theta(1-$ $z) \frac{\mathrm{e}^{\lambda z / \theta}}{(1-z)^{\mu / \theta}}$, we obtain $\frac{\mathrm{d} g_{1}(z)}{\mathrm{d} z}=\frac{(\lambda+\mu) \pi(0,1)-\theta \pi(1,1)}{-\theta(1-z) \mathrm{e}^{\lambda z / \theta}(1-z)^{-\mu / \theta}}$. By integrating this last equation, and noting that, since $f_{1}(0)=1$ and $\Pi_{1}(0)=\pi(0,1)$, then $g_{1}(0)=\pi(0,1) \neq 0$, we devise

$$
\begin{aligned}
& g_{1}(z)=\pi(0,1)-\int_{0}^{z} \frac{(\lambda+\mu) \pi(0,1)-\theta \pi(1,1)}{\theta \mathrm{e}^{\lambda x / \theta}(1-x)^{1-\mu / \theta}} \mathrm{d} x \\
& \Longrightarrow g_{1}(z)=\pi(0,1) \\
& -\frac{(\lambda+\mu) \pi(0,1)-\theta \pi(1,1)}{\theta} \int_{0}^{z} \frac{(1-x)^{\mu / \theta}}{\mathrm{e}^{\lambda x / \theta}(1-x)} \mathrm{d} x .
\end{aligned}
$$


We now aim at deriving an explicit expression for $\pi(m, 1)=$ $\left.\frac{1}{m !} \frac{\mathrm{d}^{m} \Pi_{1}(z)}{\mathrm{d} z^{m}}\right|_{z=0}$ for all $m \geq 0$. From (11) and (12) we have

$$
\begin{aligned}
& \Pi_{1}(z)=\frac{\pi(0,1) \mathrm{e}^{\frac{\lambda z}{\theta}}}{(1-z)^{\frac{\mu}{\theta}}}-\frac{((\lambda+\mu) \pi(0,1)-\theta \pi(1,1))}{\theta(1-z)^{\frac{\mu}{\theta}}} \\
& \cdot \mathrm{e}^{\frac{\lambda}{\theta}(z-1)} \cdot(-1)^{\frac{\mu}{\theta}-1}\left(\frac{\theta}{\lambda}\right)^{\frac{\mu}{\theta}} \int_{-\frac{\lambda}{\theta}}^{-\frac{\lambda}{\theta}(1-z)} y^{\frac{\mu}{\theta}-1} \mathrm{e}^{-y} \mathrm{~d} y,
\end{aligned}
$$

where we have used a change of variable $y=-\frac{\lambda}{\theta}(1-x)$ in the integral. Observe that the integral that shows up in the expression of $\Pi_{1}(z)$ is an incomplete gamma function [16, Chap. 6]. Therefore, since $\mu / \theta>0$,

$$
\int_{-\frac{\lambda}{\theta}}^{-\frac{\lambda}{\theta}(1-z)} y^{\frac{\mu}{\theta}-1} \mathrm{e}^{-y} \mathrm{~d} y=\left(-\frac{\lambda}{\theta}\right)^{\frac{\mu}{\theta}} \sum_{i=0}^{\infty} \frac{\left(\frac{\lambda}{\theta}\right)^{i}\left((1-z)^{i+\frac{\mu}{\theta}}-1\right)}{i !\left(\frac{\mu}{\theta}+i\right)} .
$$

Before deriving the probabilities $\pi(m, 1)$ for all $m \in \mathbb{N}_{0}$, note that $\Pi_{1}(z)$ is not well defined in $z=1$, and therefore we force $\lim _{z \rightarrow 1} \Pi_{1}(z)$ to be a $0 / 0$ type of indeterminate. By letting the limit as $z \rightarrow 1$ of the numerator in (13) be 0 we obtain the condition

$$
\pi(0,1) \mathrm{e}^{\lambda / \theta}-\left(\frac{\lambda+\mu}{\theta} \pi(0,1)-\pi(1,1)\right) \sum_{i=0}^{\infty} \frac{\left(\frac{\lambda}{\theta}\right)^{i}}{i !\left(\frac{\mu}{\theta}+i\right)}=0 .
$$

Solving the latter equation we obtain the explicit expression of $\pi(1,1)$ with respect to $\pi(0,1)$, namely

$$
\pi(1,1)=a_{1} \pi(0,1), \quad a_{1}=\frac{\lambda+\mu}{\theta}-\frac{\mathrm{e}^{\lambda / \theta}}{\sum_{i=0}^{\infty} \frac{(\lambda / \theta)^{i}}{i !(\mu / \theta+i)}} .
$$

After substituting $\pi(1,1)=a_{1} \pi(0,1)$ and (14) in (12), from (11) and (12) we obtain

$$
\begin{aligned}
& f_{1}(z)=\mathrm{e}^{\frac{\lambda z}{\theta}}(1-z)^{-\frac{\mu}{\theta}}, \\
& g_{1}(z)=\pi(0,1)\left(1+\frac{\sum_{j=0}^{\infty} \frac{\left(\frac{\lambda}{\theta}\right)^{j}\left((1-z)^{j+\frac{\mu}{\theta}}-1\right)}{j !\left(\frac{\mu}{\theta}+j\right)}}{\sum_{i=0}^{\infty} \frac{\left(\frac{\lambda}{\theta}\right)^{i}}{i !\left(\frac{\mu}{\theta}+i\right)}}\right) .
\end{aligned}
$$

We can now proceed to compute the steady-state distribution in the busy period, that is, $\pi(m, 1)$ for all $m \geq 1$. Let us define $\ell_{i}(\mu / \theta)=\mu / \theta \cdot \ldots \cdot(\mu / \theta+i-1)$ for all $i \geq 1$ and $\ell_{0}(\mu / \theta)=1$ and note that $\pi(m, 1)=\left.\frac{1}{m !} \frac{\mathrm{d}^{m} \Pi_{1}(z)}{\mathrm{d} z^{m}}\right|_{z=0}=$ $\frac{1}{m !} \sum_{k=0}^{m}\left(\begin{array}{c}m \\ k\end{array}\right) f_{1}^{(m-k)} g_{1}^{(k)}$, where

$$
\begin{aligned}
& f_{1}^{(k)}:=\left.\frac{\mathrm{d}^{k} f_{1}(z)}{\mathrm{d} z^{k}}\right|_{z=0}=\left.\sum_{i=0}^{k}\left(\begin{array}{c}
k \\
i
\end{array}\right)\left(\frac{\lambda}{\theta}\right)^{k-i} \frac{\mathrm{e}^{\frac{\lambda z}{\theta}} \ell_{i}\left(\frac{\mu}{\theta}\right)}{(1-z)^{\frac{\mu}{\theta}+i}}\right|_{z=0} \\
& =\sum_{i=0}^{k}\left(\begin{array}{c}
k \\
i
\end{array}\right)\left(\frac{\lambda}{\theta}\right)^{k-i} \ell_{i}\left(\frac{\mu}{\theta}\right), \text { for all } k \geq 0, \\
& g_{1}^{(k)}:=\left.\frac{\mathrm{d}^{k} g_{1}(z)}{\mathrm{d} z^{k}}\right|_{z=0} \\
& =\left.\pi(0,1) \frac{\sum_{j=0}^{\infty} \frac{\left(\frac{\lambda}{\theta}\right)^{j} \ell_{k}\left(-\frac{\mu}{\theta}-j\right)(1-z)^{j+\frac{\mu}{\theta}-k}}{j !\left(\frac{\mu}{\theta}+j\right)}}{\sum_{i=0}^{\infty} \frac{\left(\frac{\lambda}{\theta}\right)^{i}}{i !\left(\frac{\mu}{\theta}+i\right)}}\right|_{z=0} \\
& =\pi(0,1) \frac{\sum_{j=0}^{\infty} \frac{\left(\frac{\lambda}{\theta}\right)^{j} \ell_{k}\left(-\frac{\mu}{\theta}-j\right)}{j !\left(\frac{\mu}{\theta}+j\right)}}{\sum_{i=0}^{\infty} \frac{\left(\frac{\lambda}{\theta}\right)^{i}}{i !\left(\frac{\mu}{\theta}+i\right)}}, \text { for all } k \geq 1,
\end{aligned}
$$

and $g_{1}^{(0)}=\pi(0,1)$. Define $a_{1}(0):=1, a_{1}(1):=a_{1}$ and $a_{1}(m):=\frac{1}{m !} \sum_{k=0}^{m}\left(\begin{array}{c}m \\ k\end{array}\right) f_{1}^{(m-k)} g_{1}^{(k)}$ for all $m \geq 2$. Then we obtain $\pi(m, 1)=a_{1}(m) \pi(0,1)$, with $a_{1}(m)$ given as in Proposition 2.

2) Steady-state distribution in the idle period: We first define the ordinary generating function that corresponds to $\pi(m, 0)$ for all $0 \leq m \leq H-1$, that is, $\Pi_{0}(z)=$ $\sum_{m=0}^{\infty} z^{m} \pi(m, 0)=\sum_{m=0}^{H-1} z^{m} \pi(m, 0)$, where by definition $\pi(m, 0)=0$ for all $m \geq H$, and recall Equation (3) for all $1 \leq m \leq H-1$. Upon multiplying Equation (3) in state $m$ with $z^{m}$, namely

$$
\begin{aligned}
z^{m}(\lambda+m \theta) \pi(m, 0)= & z^{m} \lambda \pi(m-1,0)+z^{m} \mu \pi(m, 1) \\
& +z^{m}(m+1) \theta \pi(m+1,0),
\end{aligned}
$$

and summing the latter over all $1 \leq m \leq H-1$, we then obtain

$$
\begin{aligned}
& \lambda\left(\Pi_{0}(z)-\pi(0,0)\right)+\theta z \frac{\mathrm{d} \Pi_{0}(z)}{\mathrm{d} z}=\theta\left(\frac{\mathrm{d} \Pi_{0}(z)}{\mathrm{d} z}-\pi(1,0)\right) \\
& +\lambda z\left(\Pi_{0}(z)-\pi(H-1,0)\right)+\mu \sum_{m=1}^{H-1} z^{m} \pi(m, 1) .
\end{aligned}
$$

Using (3) in the case $m=0$, that is, $\lambda \pi(0,0)-\theta \pi(1,0)=$ $\mu \pi(0,1)$, and after some algebra, we derive

$$
\begin{aligned}
& -\frac{\lambda}{\theta} \Pi_{0}(z)+\frac{\mathrm{d} \Pi_{0}(z)}{\mathrm{d} z} \\
& =\frac{\lambda z}{\theta(1-z)} \pi(H-1,0)-\frac{\mu}{\theta(1-z)} \sum_{m=0}^{H-1} z^{m} \pi(m, 1) .
\end{aligned}
$$

Observe in the latter equation that for $\Pi_{0}(z)$ to be well defined in $z=1$, which we know equals $\sum_{m=0}^{H-1} \pi(m, 0)<1$, the condition $\lim _{z \rightarrow 1} \lambda z \pi(H-1,0)-\mu \sum_{m=0}^{H=1} \pi(m, 1)=0$ needs to be satisfied. We then obtain the extra condition to the problem

$$
\pi(H-1,0)=\frac{\mu}{\lambda} \sum_{m=0}^{H-1} \pi(m, 1)=\frac{\mu}{\lambda} \pi(0,1) \sum_{m=0}^{H-1} a_{1}(m),
$$

with $a_{1}(m)$ as given by Proposition 2. This yields $\pi(H-$ $1,0)=a_{0}^{H}(H-1) \pi(0,1)$. To derive the expression of $\pi(m, 0)$ for all $H-2 \geq m \geq 1$, we adopt the following balance equations for all $H-2 \geq m \geq 1$, which are equivalent to those introduced in Equation (3):

$$
\begin{aligned}
\lambda \pi(m, 0)= & \theta(m+1) \pi(m+1,0)+\lambda \pi(H-1,0) \\
& -\mu \sum_{j=m+1}^{H-1} \pi(j, 1) .
\end{aligned}
$$

This equation can be solved using similar arguments as those used in Section V-A, since the first two terms on the RHS of the equation correspond to the balance equations for the case $\mu=$ $\infty$. Having noticed this, the recursion can easily be solved to obtain $a_{0}^{H}(m)$ for all $H-2 \geq m \geq 1$ as given in Proposition 2 . The calculations can be found in [15]. Finally, the expression for $a_{0}^{H}(0)$ can be devised by solving $\pi(0,0)=\frac{\mu}{\lambda} \pi(0,1)+$ $\frac{\theta}{\lambda} a_{0}^{H}(1) \pi(0,1)$. Then, we obtain $\pi(m, 0)=a_{0}^{H}(m) \pi(0,1)$. 


\section{Proof of Lemma 1}

We prove that the two conditions in Lemma 1 are satisfied by Problem P. Let $E=\mathbb{N} \cup\{0\}$, and define $h(m)=\mathrm{e}^{\epsilon m}$, then, by Definition $1 h$ is a moment function. Let us first assume $\mu=\infty$, then, the first condition is equivalent to proving that there exists $\epsilon>0$ and $M>0$ such that

$$
\sum_{\tilde{m}=0}^{\infty} q^{\phi, L}(m, \tilde{m}) \mathrm{e}^{\epsilon \tilde{m}} \leq-k_{1} \mathrm{e}^{\epsilon m}, \text { for all } m \geq M,
$$

where $q^{\phi, L}(m, \tilde{m})$ denotes the transition rate from state $m$ to $\tilde{m}$. After substitution of the corresponding values of $q^{\phi, L}(\cdot, \cdot)$, we obtain for all $m \geq M$

$$
\begin{aligned}
& \lambda\left(1-\frac{m}{L}\right) \mathrm{e}^{\epsilon(m+1)}\left(1-S^{\phi}(m)\right)+\theta m \mathrm{e}^{\epsilon(m-1)}\left(1-S^{\phi}(m)\right) \\
& +\lambda\left(1-\frac{m}{L}\right) S^{\phi}(m)+\theta m S^{\phi}(m) \\
& -\left(\lambda\left(1-\frac{m}{L}\right)+\theta m\right) \mathrm{e}^{\epsilon m} \leq-k_{1} \mathrm{e}^{\epsilon m},
\end{aligned}
$$

where the first two terms on the LHS correspond to the evolution of the Markov process when the system is passive, and the third and fourth to the active period of the server. After some algebra the latter reduces to

$$
\begin{aligned}
& \lambda\left(1-\frac{m}{L}\right)\left(\mathrm{e}^{\epsilon}-1\right)+\theta m\left(\mathrm{e}^{-\epsilon}-1\right)+\theta m S^{\phi}(m)\left(\mathrm{e}^{-\epsilon m}-\mathrm{e}^{-\epsilon}\right) \\
& +\lambda\left(1-\frac{m}{L}\right) S^{\phi}(m)\left(\mathrm{e}^{-\epsilon m}-\mathrm{e}^{\epsilon}\right) \leq-k_{1},
\end{aligned}
$$

where $\lambda\left(1-\frac{m}{L}\right)\left(\mathrm{e}^{\epsilon}-1+S^{\phi}(m)\left(\mathrm{e}^{-\epsilon m}-\mathrm{e}^{\epsilon}\right)\right)$ is upper bounded by a constant, say $\kappa$, and $\theta m\left(\mathrm{e}^{-\epsilon}-1+S^{\phi}(m)\left(\mathrm{e}^{-\epsilon m}-\right.\right.$ $\left.\left.\mathrm{e}^{-\epsilon}\right)\right) \leq 0$, hence, we can find $M$ large enough so that $\theta m\left(\mathrm{e}^{-\epsilon}-1+S^{\phi}(m)\left(\mathrm{e}^{-\epsilon m}-\mathrm{e}^{-\epsilon}\right)\right) \leq-\kappa$.

Continuity of $q^{\phi, L}(\cdot, \cdot)$ in $S^{\phi}\left(N^{\phi}(t)\right)$ and $L$, follows by construction.

Let us now assume $\mu<\infty$ and $k_{1}=\left(k_{11}, k_{12}\right)$, then the first condition reduces to finding $\epsilon>0$ and $M>0$ such that for all $m \geq M$

$$
\begin{aligned}
& \lambda\left(1-\frac{m}{L}\right) \mathrm{e}^{\epsilon(m+1)}\left(1-S^{\phi}(m)\right)+\theta m \mathrm{e}^{\epsilon(m-1)}\left(1-S^{\phi}(m)\right) \\
& +\left(\lambda\left(1-\frac{m}{L}\right)+\theta m\right) S^{\phi}(m) \\
& -\left(\lambda\left(1-\frac{m}{L}\right)+\theta m\right) \mathrm{e}^{\epsilon m} \leq-k_{11} \mathrm{e}^{\epsilon m} \\
& \mu \mathrm{e}^{\epsilon m}+\lambda\left(1-\frac{m}{L}\right) \mathrm{e}^{\epsilon(m+1)}+\theta m \mathrm{e}^{\epsilon(m-1)} \\
& -\left(\lambda\left(1-\frac{m}{L}\right)+\mu+\theta m\right) \mathrm{e}^{\epsilon m} \leq-k_{12} \mathrm{e}^{\epsilon m},
\end{aligned}
$$

where the first inequality corresponds to the state $(m, 0)$ and the second inequality to $(m, 1)$. After some algebra these two inequalities reduce to

$$
\begin{aligned}
& \lambda\left(1-\frac{m}{L}\right)\left(\mathrm{e}^{\epsilon}-1+S^{\phi}(m)\left(\mathrm{e}^{-\epsilon m}-\mathrm{e}^{\epsilon}\right)\right) \\
& \theta m\left(\mathrm{e}^{-\epsilon}-1+S^{\phi}(m)\left(\mathrm{e}^{-\epsilon m}-\mathrm{e}^{-\epsilon}\right)\right) \leq-k_{11},
\end{aligned}
$$

using the same type of arguments as for the case $\mu=\infty$ the latter is true for a big enough $M$. The second inequality writes

$$
\lambda\left(1-\frac{m}{L}\right)\left(\mathrm{e}^{\epsilon}-1\right)+\theta m\left(\mathrm{e}^{-\epsilon}-1\right) \leq-k_{12},
$$

where $\lambda\left(1-\frac{m}{L}\right)\left(\mathrm{e}^{\epsilon}-1\right)$ is upper bounded and can be made as negative as desired for a big enough $M$.

Continuity of $q^{\phi, L}(\cdot, \cdot)$ in $S^{\phi}\left(N^{\phi}(t)\right)$ and $L$, follows by construction.

\section{Proof of Proposition 1}

The $\mu=\infty$ case: We prove that $V^{\infty, L}(\cdot)$ is a nondecreasing function using the Value Iteration approach [13]. To do so we define $V_{0}^{\infty, L}(m)=0$ for all $m \leq L$. Given $g^{\infty}$ the optimal average cost, and assuming w.l.o.g $\lambda+\theta L=1$ we have for all $t$

$$
\begin{aligned}
V_{t+1}^{\infty, L}(m)= & \tilde{C} m+\min \left\{\lambda\left(1-\frac{m}{L}\right) V_{t}^{\infty, L}(\min \{m+1, L\})\right. \\
& +\theta m V_{t}^{\infty, L}\left((m-1)^{+}\right)+\theta(L-m) V_{t}^{\infty, L}(m) \\
& \left.+\lambda \frac{m}{L} V_{t}^{\infty, L}(m), C_{s}+(\lambda+\theta L) V_{t}^{\infty, L}(0)\right\} .
\end{aligned}
$$

since, in the Value Iteration approach $V_{t+1}^{\infty, L}-V_{t}^{\infty, L}=g^{\infty}$. Observe that $V_{0}^{\infty, L}(\cdot)$ is non-decreasing, and it implies that $V_{1}^{\infty, L}(m)=\tilde{C} m$, which is non-decreasing given $\tilde{C}, C_{s} \geq$ 0 . Let us now use the induction argument and assume that $V_{t}^{\infty, L}(m)$ is non-decreasing, then we want to prove that

$$
V_{t+1}^{\infty, L}(m-1) \leq V_{t+1}^{\infty, L}(m),
$$

for all $m \leq L$.

Let us denote

$$
\begin{aligned}
f_{0}^{\infty}(m)= & \tilde{C} m+\lambda\left(1-\frac{m}{L}\right) V_{t}^{\infty, L}(\min \{m+1, L\}) \\
& +\theta m V_{t}^{\infty, L}\left((m-1)^{+}\right)+\theta(L-m) V_{t}^{\infty, L}(m), \\
f_{1}^{\infty}(m)= & C_{s}+(\lambda+\theta L) V_{t}^{\infty, L}(0) .
\end{aligned}
$$

To prove (16) we argue on all possible action combinations in states $m-1$ and $m$, that is, $a \in\{(0,0),(0,1),(1,0),(1,1)\}$, where $a_{1}$ is the action taken in state $m-1$ and $a_{2}$ the action taken in state $m$. We prove that for all $a$ and $m$ (16) is satisfied. Let us start with the case $a=(1,1)$, where, (16) reduces to

$$
\tilde{C}(m-1)+f_{1}^{\infty}(m-1) \leq \tilde{C} m+f_{1}^{\infty}(m) \Longrightarrow \tilde{C} \geq 0,
$$

which is by assumption true. We proceed with case $a=(0,0)$, where (16) writes

$$
\tilde{C}(m-1)+f_{0}^{\infty}(m-1) \leq \tilde{C} m+f_{0}^{\infty}(m) .
$$

We denote $\Delta V_{t}^{\infty, L}(m)=V_{t}^{\infty, L}(m)-V_{t}^{\infty, L}\left((m-1)^{+}\right)$. Then, after some algebra the latter simplifies to

$$
\begin{aligned}
& \tilde{C}+\lambda\left(1-\frac{m}{L}\right) \Delta V_{t}^{\infty, L}(m+1) \\
& \left(\lambda \frac{m}{L}+\theta(L-m)\right) \Delta V_{t}^{\infty, L}(m)+\theta m \Delta V_{t}(m-1) \geq 0,
\end{aligned}
$$

which holds due to $\tilde{C} \geq 0$ and $V_{t}^{\infty, L}(m)$ being nondecreasing. We are left with the cases $a=(0,1)$ and $a=(1,0)$, we will prove (16) for $a=(0,1)$, the other case follows similarly. Since in state $m-1$ the optimal action is passive, we have that

$$
\tilde{C}(m-1)+f_{0}^{\infty}(m-1) \leq \tilde{C}(m-1)+f_{1}^{\infty}(m-1),
$$


and we have proven in the case $a=(1,1)$ that $\tilde{C}(m-1)+$ $f_{1}^{\infty}(m-1) \leq \tilde{C} m+f_{1}^{\infty}(m)$, hence, combining these two results we obtain

$$
\tilde{C}(m-1)+f_{0}^{\infty}(m-1) \leq \tilde{C}+f_{1}^{\infty}(m),
$$

which proves Equation (16) in the case $a=(0,1)$.

Moreover, we have that $\lim _{t \rightarrow \infty} V_{t}^{\infty, L}=V^{\infty, L}$ pointwise, and therefore $V_{t}^{\infty, L}$ being non-decreasing for all $t$ implies $V^{\infty, L}$ being non-decreasing. This last argument concludes the proof.

The $\mu<\infty$ case: We first prove that $V^{L}(\cdot, 0)$ is a nondecreasing function using the Value Iteration approach [13] on the truncated system. To do so we smooth the arrival rate such that $q(m-1, m)=\lambda\left(1-\frac{m}{L}\right)$.

1) $V^{L}(\cdot, 0)$ is non-decreasing: To prove that $V^{L}(\cdot, 0)$ is a non-decreasing function we define $V_{0}^{L}(m)=0$ for all $m \leq$ $L$. Given $g$ the optimal average cost, and assuming w.l.o.g $\lambda+\mu+\theta L=1$ the Bellman equation writes

$$
\begin{aligned}
& V_{t+1}^{L}(m, 0) \\
& =\tilde{C} m+\min \left(\lambda\left(1-\frac{m}{L}\right) V_{t}^{L}(m+1,0)\right. \\
& +\left(\lambda \frac{m}{L}+\mu+\theta(L-m)\right) V_{t}^{L}(m, 0)+\theta m V_{t}^{L}(m-1,0) \\
& \left.C_{s}+\lambda V_{t}^{L}(1,1)+\theta L V_{t}^{L}(0,1)+\mu V_{t}^{L}(0,0)\right),
\end{aligned}
$$

and

$$
\begin{aligned}
& V_{t+1}^{L}(m, 1)=\tilde{C} m+\lambda\left(1-\frac{m}{L}\right) V_{t}^{L}(m+1,1)+\mu V_{t}^{L}(m, 0) \\
& +\left(\lambda \frac{m}{L}+(L-m) \theta\right) V_{t}^{L}(m, 1)+m \theta V_{t}^{L}(m-1,1),
\end{aligned}
$$

since $V_{t+1}^{L}(m, 0)-V_{t}^{L}(m, 0)=g$.

We will first prove that for all $t, V_{t}^{L}(m, 0)$ is nondecreasing in $m$. We will argue by induction: first we show that $V_{0}^{L}(m, 0) \geq V_{0}^{L}\left(m^{\prime}, 0\right)$ for all $m \geq m^{\prime} \geq 0$ implies that $V_{1}^{L}(m, 0) \geq V_{1}^{L}\left(m^{\prime}, 0\right)$, and later on we will prove that assuming that $V_{t}^{L}(m, a)$ is non-decreasing in $m$, implies $V_{t+1}^{L}(m, 1)$ to be non-decreasing. By definition $V^{L}(m, a)$ is the asymptotic difference in total reward from starting at state $m$ instead of starting at the reference state which, without loss of generality, we set at 0 . We choose $V_{0}^{L}(m, a)=0$ for all $m \geq 0$ and $a \in\{0,1\}$, then, from (17) and (18) we obtain $V_{1}^{L}(m, a)=\tilde{C} m$. Since $\tilde{C}>0, V_{1}^{L}(m, a) \geq V_{1}^{L}(m-1, a)$ for all $m \geq 1$. We now assume that $V_{t}^{L}(m, 0)$ is non-decreasing, and we prove that $V_{t+1}^{L}(m, 0) \geq V_{t+1}^{L}(m-1,0)$ for all $L \geq m \geq 1$, that is, after substitution of (17), equivalent to proving

$$
\begin{aligned}
& \tilde{C} m+\min \left(\lambda\left(1-\frac{m}{L}\right) V_{t}^{L}(m+1,0)+\theta m V_{t}^{L}(m-1,0)\right. \\
& +\left(\lambda \frac{m}{L}+\mu+\theta(L-m)\right) V_{t}^{L}(m, 0), C_{s}+\lambda V_{t}^{L}(1,1) \\
& \left.+\theta N V_{t}^{L}(0,1)+\mu V_{t}^{L}(0,0)\right) \\
& \geq \tilde{C}(m-1)+\min \left(\lambda\left(1-\frac{m-1}{L}\right) V_{t}^{L}(m, 0)\right. \\
& +\left(\lambda \frac{m-1}{L}+\mu+\theta(L-m+1)\right) V_{t}^{L}(m-1,0) \\
& +\theta(m-1) V_{t}^{L}\left((m-2)^{+}, 0\right), C_{s}+\lambda V_{t}^{L}(1,1)+\theta L V_{t}^{L}(0,1) \\
& \left.+\mu V_{t}^{L}(0,0)\right) .
\end{aligned}
$$

We will now prove that Inequality (19) is satisfied for all possible action combinations in states $m$ and $m-1$. Let us first assume that in both $m$ and $m-1$ passive action is optimal, then (19) reduces to

$$
\begin{aligned}
& \tilde{C} m+\lambda\left(1-\frac{m}{L}\right) V_{t}^{L}(m+1,0)+\theta m V_{t}^{L}(m-1,0) \\
& +\left(\lambda \frac{m}{L}+\mu+\theta(L-m)\right) V_{t}^{L}(m, 0) \\
& \geq \tilde{C}(m-1)+\lambda\left(1-\frac{m-1}{L}\right) V_{t}^{L}(m, 0) \\
& +\left(\lambda \frac{m-1}{L}+\mu+\theta(L-m+1)\right) V_{t}^{L}(m-1,0) \\
& +\theta(m-1) V_{t}^{L}\left((m-2)^{+}, 0\right),
\end{aligned}
$$

which after some calculations writes

$$
\begin{aligned}
& \tilde{C}+\lambda\left(1-\frac{m}{L}\right) \Delta V_{t}^{L}(m+1,0)+\theta(m-1) \Delta V_{t}^{L}(m-1,0) \\
& +\left(\lambda \frac{m-1}{L}+\mu+\theta(L-m)\right) \Delta V_{t}^{L}(m, 0) \geq 0,
\end{aligned}
$$

for all $L \geq m \geq 1$ and $\Delta V_{t}^{L}(m, 0)=V_{t}^{L}(m, 0)-V_{t}^{L}((m-$ $\left.1)^{+}, 0\right)$. Due to $\Delta V_{t}^{L}(m, 0) \geq 0$ for all $L \geq m \geq 0$, this last inequality is satisfied. We now prove (19) for the case in which active action is optimal in both $m$ and $m-1$, then, Inequality (19) reduces to

$$
\begin{aligned}
& \tilde{C} m+C_{s}+\lambda V_{t}^{L}(1,1)+\theta L V_{t}^{L}(0,1)+\mu V_{t}^{L}(0,0) \\
& \geq \tilde{C}(m-1)+C_{s}+\lambda V_{t}^{L}(1,1)+\theta L V_{t}^{L}(0,1)+\mu V_{t}^{L}(0,0),
\end{aligned}
$$

which simplifies to $\tilde{C} \geq 0$, and hence (19) is satisfied. Let us now proceed with assuming that passive action is optimal in $m$ and active in $m-1$, then the following holds,

$$
\begin{aligned}
& \tilde{C} m+\lambda\left(1-\frac{m}{L}\right) V_{t}^{L}(m+1,0)+\theta m V_{t}^{L}(m-1,0) \\
& +\left(\lambda \frac{m}{L}+\mu+\theta(L-m)\right) V_{t}^{L}(m, 0) \\
& \geq \tilde{C}(m-1)+\min \left(\lambda\left(1-\frac{m-1}{L}\right) V_{t}^{L}(m, 0)\right. \\
& +\left(\lambda \frac{m-1}{L}+\mu+\theta(L-m+1)\right) V_{t}^{L}(m-1,0) \\
& \geq \tilde{C}(m-1)+C_{s}+\lambda V_{t}^{L}(1,1)+\theta L V_{t}^{L}(0,1)+\mu V_{t}^{L}(0,0),
\end{aligned}
$$


where the first inequality has been proven above, and the second inequality follows from the fact that active action is optimal in $m$. Therefore, Inequality (19) holds in case active is optimal in $m-1$ and passive in $m$. We are left with the proof in the case where the optimal action is active in $m$ and passive in $m-1$. We have

$$
\begin{aligned}
& \tilde{C} m+C_{s}+\lambda V_{t}^{L}(1,1)+\theta L V_{t}^{L}(0,1)+\mu V_{t}^{L}(0,0) \\
& \geq \tilde{C}(m-1)+C_{s}+\lambda V_{t}^{L}(1,1)+\theta L V_{t}^{L}(0,1)+\mu V_{t}^{L}(0,0) \\
& \geq \tilde{C}(m-1)+\min \left(\lambda\left(1-\frac{m-1}{L}\right) V_{t}^{L}(m, 0)\right. \\
& +\left(\lambda \frac{m-1}{L}+\mu+\theta(L-m+1)\right) V_{t}^{L}(m-1,0) \\
& +\theta(m-1) V_{t}^{L}\left((m-2)^{+}, 0\right),
\end{aligned}
$$

where the first inequality has been proven above and the second inequality follows from the fact that passive action in optimal in state $m-1$. Therefore, Inequality (19) holds.

We have therefore proven that for any $t, V_{t}(m, 0)$ is nondecreasing in $m$. And $V_{t}^{L}(m, 0) \rightarrow V^{L}(m, 0)$ point-wise, then, $V^{L}(m, 0)$ is non-decreasing.

Let us now prove $f_{t}^{L}(m, 0)-f_{t}^{L}(m, 1) \leq f_{t}^{L}\left(m^{\prime}, 0\right)-$ $f_{t}^{L}\left(m^{\prime}, 1\right)$ where

$$
\begin{aligned}
f_{t}^{L}(m, 0)= & \lambda\left(1-\frac{m}{L}\right) V_{t}^{L}(m+1,0)+\theta m V_{t}^{L}(m-1,0) \\
& +\left(\lambda \frac{m}{L}+\mu+\theta(L-m)\right) V_{t}^{L}(m, 0), \\
f_{t}^{L}(m, 1)= & \tilde{C} m+C_{s}+\lambda V_{t}^{L}(1,1)+\theta L V_{t}^{L}(0,1) \\
& +\mu V_{t}^{L}(0,0) .
\end{aligned}
$$

2) $f_{t}^{L}(m, 0)-f_{t}^{L}(m, 1) \leq f_{t}^{L}\left(m^{\prime}, 0\right)-f_{t}^{L}\left(m^{\prime}, 1\right)$ for all $m^{\prime} \geq m$ : . Substituting the expression of $f_{t}^{L}(m, a)$ the inequality reduces to

$$
\begin{aligned}
& \tilde{C}\left(m^{\prime}-m\right) \leq \tilde{C}\left(m^{\prime}-m\right)+\lambda\left(1-\frac{m^{\prime}}{L}\right) V_{t}^{L}\left(m^{\prime}+1,0\right) \\
& +\left(\lambda \frac{m^{\prime}}{L}+\mu+\theta\left(L-m^{\prime}\right)\right) V_{t}^{L}\left(m^{\prime}, 0\right)+\theta m^{\prime} V_{t}^{L}\left(m^{\prime}-1,0\right) \\
& -\left(\lambda\left(1-\frac{m}{L}\right) V_{t}^{L}(m+1,0)\right. \\
& \left.+\left(\lambda \frac{m}{L}+\mu+\theta(L-m)\right) V_{t}^{L}(m, 0)+\theta m V_{t}^{L}(m-1,0)\right) .
\end{aligned}
$$

Let us now define $m^{\prime}=m+u$, with $u \geq 1$, and then the latter inequality writes

$$
\begin{aligned}
0 \leq & \lambda\left(1-\frac{m^{\prime}}{L}\right)\left(V_{t}^{L}\left(m^{\prime}+1,0\right)-V_{t}^{L}(m+1,0)\right) \\
& -\lambda \frac{u}{L}\left(V_{t}^{L}(m+1,0)-V_{t}^{L}(m, 0)\right) \\
& +\lambda \frac{m^{\prime}}{L}\left(V_{t}^{L}\left(m^{\prime}, 0\right)-V_{t}^{L}(m, 0)\right) \\
& +\mu\left(V_{t}^{L}\left(m^{\prime}, 0\right)-V_{t}^{L}(m, 0)\right) \\
& +\theta m\left(V_{t}^{L}\left(m^{\prime}-1,0\right)-V_{t}^{L}(m-1,0)\right) \\
& +\theta u\left(V_{t}^{L}\left(m^{\prime}-1,0\right)-V_{t}^{L}(m, 0)\right)
\end{aligned}
$$

which is satisfied due to $V_{t}^{L}(m, 0)$ being non-decreasing.
E. Expression of $\pi_{m}$ in the case $\mu=\infty$

We solve the balance equations in Section $\mathrm{V}-\mathrm{A}$, that is,

$$
\begin{aligned}
\pi_{m}= & (m+1) \frac{\theta}{\lambda} \pi_{m+1}+\pi_{H-1} \\
= & \pi_{H-1}+(m+1) \frac{\theta}{\lambda}\left((m+2) \frac{\theta}{\lambda} \pi_{m+2}+\pi_{H-1}\right) \\
= & \pi_{H-1}\left(1+\frac{\theta}{\lambda}(m+1)\right)+\pi_{m+2}\left(\frac{\theta}{\lambda}\right)^{2}(m+1)(m+2) \\
= & \pi_{H-1}\left(1+\frac{\theta}{\lambda}(m+1)+\left(\frac{\theta}{\lambda}\right)^{2}(m+1)(m+2)\right) \\
& +\pi_{m+3}\left(\frac{\theta}{\lambda}\right)^{3}(m+1)(m+2)(m+3) \\
= & \ldots \\
= & \pi_{H-1}\left(1+\sum_{i=1}^{H-1-m}\left(\frac{\theta}{\lambda}\right)^{i} \frac{(m+i) !}{m !}\right)
\end{aligned}
$$

F. How to deduce the expression of $a_{0}^{H}(m)$

Recall Equation (3) and note that one can equivalently write for all $H-2 \geq m \geq 1$

$$
\begin{aligned}
\lambda \pi(m, 0)= & \theta(m+1) \pi(m+1,0)+\lambda \pi(H-1,0) \\
& -\mu \sum_{j=m+1}^{H-1} \pi(j, 1) .
\end{aligned}
$$

Then,

$$
\begin{aligned}
& \pi(m, 0)=\frac{\theta(m+1)}{\lambda} \pi(m+1,0)+\pi(H-1,0) \\
& -\frac{\mu}{\lambda} \sum_{j=m+1}^{H-1} \pi(j, 1) \\
& =\frac{\theta(m+1)}{\lambda}\left(\frac{\theta(m+2)}{\lambda} \pi(m+2,0)+\pi(H-1,0)\right. \\
& \left.-\frac{\mu}{\lambda} \sum_{j=m+2}^{H-1} \pi(j, 1)\right)+\pi(H-1,0) \\
& -\frac{\mu}{\lambda} \sum_{j=m+1}^{H-1} \pi(j, 1) \\
& =\frac{\theta^{2}}{\lambda^{2}}(m+1)(m+2) \pi(m+2,0) \\
& +\left(1+\frac{\theta}{\lambda}(m+1)\right) \pi(H-1,0) \\
& -\frac{\mu}{\lambda} \pi(m+1,1)-\frac{\mu}{\lambda} \sum_{j=m+2}^{H-1}\left(1+\frac{\theta(m+1)}{\lambda}\right) \pi(j, 1) \\
& =\ldots \\
& =\pi(H-1,0) \sum_{i=0}^{H-1-m}\left(\frac{\theta}{\lambda}\right)^{i} \frac{(m+i) !}{m !} \\
& -\frac{\mu}{\lambda} \sum_{j=m+1}^{H-1} \pi(r, 1) \sum_{i=0}^{r-m-1}\left(\frac{\theta}{\lambda}\right)^{i} \frac{(m+i) !}{m !} .
\end{aligned}
$$

This last expression is valid for all $m \geq 1$. 


\section{G. Proof of Proposition 3}

We aim at proving that for all $H^{\prime} \neq H$

$$
\tilde{C} \mathbb{E}\left(N^{H}\right)+C_{s} P_{b}^{H} \leq \tilde{C} \mathbb{E}\left(N^{H^{\prime}}\right)+C_{s} P_{b}^{H^{\prime}} .
$$

We present here the proof in the case $H^{\prime}<H$, the other case can be done similarly. By assumption we have for all $H \geq 1$, $\alpha(H-1) \leq \alpha(H)$, then

$$
\begin{aligned}
& \frac{\mathbb{E}\left(N^{H-1}\right)-\mathbb{E}\left(N^{H-2}\right)}{P_{b}^{H-2}-P_{b}^{H-1}} \leq \frac{\mathbb{E}\left(N^{H}\right)-\mathbb{E}\left(N^{H-1}\right)}{P_{b}^{H-1}-P_{b}^{H}} \\
& \Longrightarrow\left(\mathbb{E}\left(N^{H-1}\right)-\mathbb{E}\left(N^{H-2}\right)\right)\left(P_{b}^{H-1}-P_{b}^{H}\right) \\
& \leq\left(\mathbb{E}\left(N^{H}\right)-\mathbb{E}\left(N^{H-1}\right)\right)\left(P_{b}^{H-2}-P_{b}^{H-1}\right) .
\end{aligned}
$$

In the latter inequality we sum and subtract $\mathbb{E}\left(N^{H}\right)\left(P_{b}^{H-1}\right.$ $\left.P_{b}^{H}\right)$ on the left hand side, that is,

$$
\begin{aligned}
& \left(\mathbb{E}\left(N^{H-1}\right)-\mathbb{E}\left(N^{H}\right)+\mathbb{E}\left(N^{H}\right)-\mathbb{E}\left(N^{H-2}\right)\right)\left(P_{b}^{H-1}-P_{b}^{H}\right) \\
& \leq\left(\mathbb{E}\left(N^{H}\right)-\mathbb{E}\left(N^{H-1}\right)\right)\left(P_{b}^{H-2}-P_{b}^{H-1}\right),
\end{aligned}
$$

after some algebra this last inequality reduces to

$$
\frac{\mathbb{E}\left(N^{H}\right)-\mathbb{E}\left(N^{H-2}\right)}{P_{b}^{H-2}-P_{b}^{H}} \leq \frac{\mathbb{E}\left(N^{H}\right)-\mathbb{E}\left(N^{H-1}\right)}{P_{b}^{H-1}-P_{b}^{H}} \leq C_{s} .
$$

Similarly, one can prove

$$
\alpha(H-1) \leq \frac{\mathbb{E}\left(N^{H}\right)-\mathbb{E}\left(N^{H-2}\right)}{P_{b}^{H-2}-P_{b}^{H}} .
$$

We now make the following induction assumption for a given $a$

$$
\alpha(H-a+1) \leq \frac{\mathbb{E}\left(N^{H}\right)-\mathbb{E}\left(N^{H-a}\right)}{P_{b}^{H-a}-P_{b}^{H}} \leq C_{s} .
$$

By assumption on the statement we have $\alpha(H-a) \leq \alpha(H-$ $a+1)$, hence from the latter equation we obtain

$$
\begin{aligned}
& \alpha(H-a) \leq \frac{\mathbb{E}\left(N^{H}\right)-\mathbb{E}\left(N^{H-a}\right)}{P_{b}^{H-a}-P_{b}^{H}} \\
& \Longrightarrow\left(\mathbb{E}\left(N^{H-a}\right)-\mathbb{E}\left(N^{H-a-1}\right)\right)\left(P_{b}^{H-a}-P_{b}^{H}\right) \\
& \leq\left(\mathbb{E}\left(N^{H}\right)-\mathbb{E}\left(N^{H-a}\right)\right)\left(P_{b}^{H-a-1}-P_{b}^{H-a}\right),
\end{aligned}
$$

adding and subtracting $\mathbb{E}\left(N^{H}\right)\left(P_{b}^{H-a}-P_{b}^{H}\right)$ on the left hand side, and after some algebra, we obtain

$$
\frac{\mathbb{E}\left(N^{H}\right)-\mathbb{E}\left(N^{H-a-1}\right)}{P_{b}^{H-a-1}-P_{b}^{H}} \leq \frac{\mathbb{E}\left(N^{H}\right)-\mathbb{E}\left(N^{H-a}\right)}{P_{b}^{H-a}-P_{b}^{H}} \leq C_{s} .
$$

From the latter we observe that

$$
\begin{aligned}
& \left(\mathbb{E}\left(N^{H}\right)-\mathbb{E}\left(N^{H-a-1}\right)\right)\left(P_{b}^{H-a}-P_{b}^{H}+P_{b}^{H-a-1}-P_{b}^{H-a-1}\right) \\
& \leq\left(\mathbb{E}\left(N^{H}\right)-\mathbb{E}\left(N^{H-a}\right)\right)\left(P_{b}^{H-a-1}-P_{b}^{H}\right),
\end{aligned}
$$

which after some algebra reduces to

$$
\alpha(H-a) \leq \frac{\mathbb{E}\left(N^{H}\right)-\mathbb{E}\left(N^{H-a-1}\right)}{P_{b}^{H-a-1}-P_{b}^{H}} .
$$

The latter together with (20) gives

$$
\alpha(H-a) \leq \frac{\mathbb{E}\left(N^{H}\right)-\mathbb{E}\left(N^{H-a-1}\right)}{P_{b}^{H-a-1}-P_{b}^{H}} \leq C_{s},
$$

which concludes the induction. For all $0 \leq a \leq H-1$ denote $H^{\prime}=H-1-a$. We have proven that for all $H^{\prime}<H$

$$
\begin{aligned}
& \frac{\mathbb{E}\left(N^{H}\right)-\mathbb{E}\left(N^{H^{\prime}}\right)}{P_{b}^{H^{\prime}}-P_{b}^{H}} \leq C_{s} \\
& \Longrightarrow \tilde{C} \mathbb{E}\left(N^{H}\right)+C_{s} P_{b}^{H} \leq \tilde{C} \mathbb{E}\left(N^{H^{\prime}}\right)+C_{s} P_{b}^{H^{\prime}} .
\end{aligned}
$$

Which concludes the proof.

\section{H. Proof of Lemma 2}

Let us first proof that $P_{b}^{H}$ is non-increasing. It suffices to prove $\pi_{H-1}^{H} \leq \pi_{H-2}^{H-1}$ for all $H \geq 2$. This inequality writes

$$
\begin{aligned}
& \sum_{m=0}^{H-2} \sum_{i=0}^{H-2-m}\left(\frac{\theta}{\lambda}\right)^{i} \frac{(m+i) !}{m !} \leq \sum_{m=0}^{H-1} \sum_{i=0}^{H-1-m}\left(\frac{\theta}{\lambda}\right)^{i} \frac{(m+i) !}{m !} \\
& \Longleftrightarrow 0 \leq \sum_{m=0}^{H-1}\left(\frac{\theta}{\lambda}\right)^{H-1-m} \frac{(H-1) !}{m !} .
\end{aligned}
$$

The RHS is positive for all $H \geq 2$ and hence $\pi_{H-1}^{H} \leq \pi_{H-2}^{H-1}$. Having proven that $\pi_{H-1}^{H}$ is non-increasing in $H$, we now proceed to prove the convexity of $P_{b}^{H}$. One can easily prove that convexity of $P_{b}^{H}$ is implied by $\pi_{H-1}^{H}-\pi_{H}^{H+1} \leq \pi_{H-2}^{H-1}-$ $\pi_{H-1}^{H}$, which after substitution of the corresponding values reduces to

$\frac{\sum_{m=0}^{H+1} \sum_{i=0}^{H+1-m}\left(\frac{\theta}{\lambda}\right)^{i} \frac{(m+i) !}{m !}-\sum_{m=0}^{H} \sum_{i=0}^{H-m}\left(\frac{\theta}{\lambda}\right)^{i} \frac{(m+i) !}{m !}}{\sum_{m=0}^{H+1} \sum_{i=0}^{H+1-m}\left(\frac{\theta}{\lambda}\right)^{i} \frac{(m+i) !}{m !}}$

$\leq \frac{\sum_{m=0}^{H} \sum_{i=0}^{H-m}\left(\frac{\theta}{\lambda}\right)^{i} \frac{(m+i) !}{m !}-\sum_{m=0}^{H-1} \sum_{i=0}^{H-1-m}\left(\frac{\theta}{\lambda}\right)^{i} \frac{(m+i) !}{m !}}{\sum_{m=0}^{H-1} \sum_{i=0}^{H-1-m}\left(\frac{\theta}{\lambda}\right)^{i} \frac{(m+i) !}{m !}}$.

After some algebra the latter reduces to

$$
\begin{aligned}
& \left(\sum_{m=0}^{H+1}\left(\frac{\theta}{\lambda}\right)^{H+1-m} \frac{(H+1) !}{m !}\right) \sum_{m=0}^{H-1} \sum_{i=0}^{H-1-m}\left(\frac{\theta}{\lambda}\right)^{i} \frac{(m+i) !}{m !} \\
& \leq\left(\sum_{m=0}^{H}\left(\frac{\theta}{\lambda}\right)^{H-m} \frac{H !}{m !}\right) \sum_{m=0}^{H+1} \sum_{i=0}^{H+1-m}\left(\frac{\theta}{\lambda}\right)^{i} \frac{(m+i) !}{m !},
\end{aligned}
$$

which is satisfied for all $H$. Hence, $P_{b}^{H}$ is convex in $H$.

Let us now prove that $\alpha(H)$ as defined in Proposition 3 is non-decreasing, that is,

$$
\begin{aligned}
& \alpha(H):=\tilde{C} \frac{\mathbb{E}\left(N^{H}\right)-\mathbb{E}\left(N^{H-1}\right)}{P_{b}^{H-1}-P_{b}^{H}} \\
& =\frac{\sum_{m=0}^{H-1} m \pi_{m}^{H}-\sum_{m=0}^{H-2} m \pi_{m}^{H-1}}{P_{b}^{H-1}-P_{b}^{H}} \\
& =\frac{\sum_{m=0}^{H-1} m\left(\sum_{i=0}^{H-1-m}\left(\frac{\theta}{\lambda}\right)^{i} \frac{(m+i) !}{m !}\right) \pi_{H-1}^{H}}{P_{b}^{H-1}-P_{b}^{H}} \\
& \quad-\frac{\sum_{m=0}^{H-2} m\left(\sum_{i=0}^{H-2-m}\left(\frac{\theta}{\lambda}\right)^{i} \frac{(m+i) !}{m !}\right) \pi_{H-2}^{H-1}}{P_{b}^{H-1}-P_{b}^{H}} .
\end{aligned}
$$


The latter after some algebra writes

$$
\begin{aligned}
\alpha(H)= & \frac{\sum_{m=0}^{H-1} m\left(\frac{\theta}{\lambda}\right)^{H-1-m} \frac{(H-1) !}{m !} \pi_{H-1}^{H}}{P_{b}^{H-1}-P_{b}^{H}} \\
& +\sum_{m=0}^{H-2} m \sum_{i=0}^{H-2-m}\left(\frac{\theta}{\lambda}\right)^{i} \frac{(m+i) !}{m !}\left(\frac{\pi_{H-1}^{H}-\pi_{H-2}^{H-1}}{P_{b}^{H-1}-P_{b}^{H}}\right) .
\end{aligned}
$$

We now aim at proving $\alpha(H) \leq \alpha(H+1)$ for all $H$, that is if

$$
\begin{aligned}
& \frac{\sum_{m=0}^{H-1} m\left(\frac{\theta}{\lambda}\right)^{H-1-m} \frac{(H-1) !}{m !} \pi_{H-1}^{H}}{P_{b}^{H-1}-P_{b}^{H}} \\
& +\sum_{m=0}^{H-2} m \sum_{i=0}^{H-2-m}\left(\frac{\theta}{\lambda}\right)^{i} \frac{(m+i) !}{m !}\left(\frac{\pi_{H-1}^{H}-\pi_{H-2}^{H-1}}{P_{b}^{H-1}-P_{b}^{H}}\right) \\
& \leq \frac{\sum_{m=0}^{H} m\left(\frac{\theta}{\lambda}\right)^{H-m} \frac{H !}{m !} \pi_{H}^{H+1}}{P_{b}^{H}-P_{b}^{H+1}} \\
& +\sum_{m=0}^{H-1} m \sum_{i=0}^{H-1-m}\left(\frac{\theta}{\lambda}\right)^{i} \frac{(m+i) !}{m !}\left(\frac{\pi_{H}^{H+1}-\pi_{H-1}^{H}}{P_{b}^{H}-P_{b}^{H+1}}\right),
\end{aligned}
$$

The second term in the LHS of the inequality being less than or equal to the second term in the RHS of the inequality follows from $P_{b}^{H}$ and $\pi_{H-1}^{H}$ being convex non-increasing functions in $H$. We are therefore left to proof

$$
\begin{aligned}
& \sum_{m=0}^{H-1} m\left(\frac{\theta}{\lambda}\right)^{H-1-m} \frac{(H-1) !}{m !} \pi_{H-1}^{H} \\
& \leq \sum_{m=0}^{H} m\left(\frac{\theta}{\lambda}\right)^{H-m} \frac{H !}{m !} \pi_{H}^{H+1} .
\end{aligned}
$$

This concludes the proof.

\section{Proof of Lemma 3}

To prove that $P_{b}^{H}$ is non-increasing it suffices to prove $\pi^{H}(0,1) \geq \pi^{H+1}(0,1)$. Observe that $\pi^{H}(0,1) \geq \pi^{H+1}(0,1)$ is implied by $\sum_{m=0}^{H-1} a_{0}^{H}(m) \leq \sum_{m=0}^{H} a_{0}^{H+1}(m)$. Furthermore, after some algebra one can obtain that

$$
\sum_{m=0}^{H} a_{0}^{H+1}(m)=\sum_{m=0}^{H} a_{0}^{H}(m)+\frac{\mu}{\lambda} \sum_{r=0}^{H} a_{1}(r)\left(\frac{\theta}{\lambda}\right)^{H-m} \frac{H !}{m !},
$$

therefore

$$
\begin{aligned}
& 0 \leq \sum_{m=0}^{H} a_{0}^{H+1}(m)-\sum_{m=0}^{H-1} a_{0}^{H}(m) \\
& \Longleftrightarrow 0 \leq a_{0}^{H}(H)+\frac{\mu}{\lambda} \sum_{r=0}^{H} a_{1}(r)\left(\frac{\theta}{\lambda}\right)^{H-m} \frac{H !}{m !} .
\end{aligned}
$$

The last inequality is satisfied since $a_{0}^{H}(m)>0$ for all $m$. 